\title{
Hydrometeorological data from Marmot Creek Research Basin, Canadian Rockies
}

\author{
Xing Fang, John W. Pomeroy, Chris M. DeBeer, Phillip Harder, and Evan Siemens \\ Centre for Hydrology and Global Institute for Water Security, University of Saskatchewan, \\ Saskatoon, S7N 1K2, Canada \\ Correspondence: Xing Fang (xing.fang@usask.ca)
}

Received: 26 September 2018 - Discussion started: 12 October 2018

Revised: 1 March 2019 - Accepted: 18 March 2019 - Published: 10 April 2019

\begin{abstract}
Meteorological, snow survey, streamflow, and groundwater data are presented from Marmot Creek Research Basin, Alberta, Canada. The basin is a $9.4 \mathrm{~km}^{2}$, alpine-montane forest headwater catchment of the Saskatchewan River basin that provides vital water supplies to the Prairie Provinces of Canada. It was heavily instrumented, experimented upon, and operated by several federal government agencies between 1962 and 1986, during which time its main and sub-basin streams were gauged, automated meteorological stations at multiple elevations were installed, groundwater observation wells were dug and automated, and frequent manual measurements of snow accumulation and ablation and other weather and water variables were made. Over this period, mature evergreen forests were harvested in two sub-basins, leaving large clear cuts in one basin and a "honeycomb" of small forest clearings in another basin. Whilst meteorological measurements and sub-basin streamflow discharge weirs in the basin were removed in the late 1980s, the federal government maintained the outlet streamflow discharge measurements and a nearby high-elevation meteorological station, and the Alberta provincial government maintained observation wells and a nearby fire weather station. Marmot Creek Research Basin was intensively re-instrumented with 12 automated meteorological stations, four sub-basin hydrometric sites, and seven snow survey transects starting in 2004 by the University of Saskatchewan Centre for Hydrology. The observations provide detailed information on meteorology, precipitation, soil moisture, snowpack, streamflow, and groundwater during the historical period from 1962 to 1987 and the modern period from 2005 to the present time. These data are ideal for monitoring climate change, developing hydrological process understanding, evaluating process algorithms and hydrological, cryospheric, or atmospheric models, and examining the response of basin hydrological cycling to changes in climate, extreme weather, and land cover through hydrological modelling and statistical analyses. The data presented are publicly available from Federated Research Data Repository (https://doi.org/10.20383/101.09, Fang et al., 2018).
\end{abstract}

\section{Introduction}

The eastern slopes of the Canadian Rocky Mountains form the headwaters of the Saskatchewan River basin (SRB), whose water supplies are vital to domestic, agricultural, and industrial users in the Canadian Prairie Provinces. These mountain headwaters occupy about $12.6 \%$ of total drainage area but generate $87 \%$ of total water yield in the SRB (Redmond, 1964). Recognising the importance of these headwaters, the Eastern Rocky Mountain Forest Conservation Act was passed in 1947, which aimed to conserve and protect the Saskatchewan River headwaters (Neill, 1980; Rothwell et al., 2016). The Eastern Slopes (Alberta) Watershed Research Program (AWRP) was created in 1960 to investigate relationships between forest, soil, climate, and water and to examine the impacts of commercial timber harvesting practices on basin water yield and water quality (Jeffrey, 1965; Kirby and Ogilvy, 1969). This program was a collaborative effort between several provincial and federal government agencies to establish experimental watersheds in the headwaters, one of which was the establishment of what was then called the 
"Marmot Creek Experimental Watershed" during 1961-1962 (Rothwell et al., 2016). This later became the University of Saskatchewan-operated "Marmot Creek Research Basin" (MCRB) by which it is referred to in this paper.

During the historical period of 1962-1986, a paired-basin experiment devised by the Canadian Forestry Service (CFS) explored the effects of forest cutting on snow accumulation and water yield in MCRB. Two types of forest clearing experiment were conducted in the sub-alpine spruce-fir forest part of the basin (Fig. 1): six large "commercial" forest cut blocks were harvested in the Cabin Creek sub-basin during 1971-1972 and a "honeycomb" of numerous small circular clearings, each 12 to $18 \mathrm{~m}$ in diameter, were harvested in the Twin Creek sub-basin during 1977-1979, with Middle Creek left intact as a control sub-basin (Rothwell et al., 2016). Snow accumulation increased by $21 \%$ in the large forest cutting blocks (Swanson et al., 1986) and $28 \%$ in the small forest clearings compared to under adjacent intact forest canopies (Swanson and Golding, 1982). Overall, there was no statistically significant change in streamflow that could be associated with the forestry manipulations (Harder et al., 2015). Several other studies were carried out in parallel to the forest clearing experiments. Investigations on soil water storage and soil temperature in relation to snow accumulation and melt, forest, and slope orientation were conducted at several sites in MCRB and provided some early understanding of infiltration and runoff in the basin (Harlan, 1969; Hillman and Golding, 1981). Extensive field campaigns throughout MCRB produced detailed descriptions of soils (Beke, 1969) and surficial geology (Stevenson, 1967). Additional studies were undertaken to assess the basin's meteorology (Munn and Storr, 1967; Storr, 1967, 1973). Most hydrometeorological observations in MCRB ceased after 1986 due to the opening of the adjacent Nakiska Ski Resort in the 1986-1987 ski season and subsequent hosting of 1988 Winter Olympic Games; only streamflow measurements at the main outlet by Environment and Climate Change Canada (ECCC), and groundwater measurements by Alberta Environment and Parks (AEP), were continued, though a high-elevation weather station was established on Centennial Ridge by ECCC, and Alberta Agriculture and Forestry maintained a nearby valley-bottom weather station (Rothwell et al., 2016).

After the Olympics, research activities in MCRB were minimal until 2004 when the research basin was reactivated by the University of Saskatchewan with the help of the University of Calgary and ECCC. Wide-ranging research has been conducted since then to improve the understanding of the impact of forest canopy and forest clearings on snow accumulation and snowmelt energetics (Ellis and Pomeroy, 2007; Essery et al., 2008; Pomeroy et al., 2009; Ellis et al., 2013; Musselman and Pomeroy, 2017), slope and aspect controls on snow accumulation and melt (DeBeer and Pomeroy, 2009; Ellis et al., 2011; Marsh et al., 2012), blowing snow and sublimation in the alpine treeline environ- ment with respect to local wind and topography (MacDonald et al., 2010), alpine snowmelt runoff generation (DeBeer and Pomeroy, 2017), hillslope hydrology of the forest organic layer (Keith et al., 2010), and precipitation phase partitioning (Harder and Pomeroy, 2013). MCRB has also been the site of instrument or methodology development, from an early airborne lidar snow depth measurement (Hopkinson et al., 2012) to acoustic measurements of snow (Kinar and Pomeroy, 2009) as well as early telescope-based snow surveys (Kinar and Pomeroy, 2015). Utilising the Cold Regions Hydrological Modelling platform (CRHM) these advances have been synthesised into a physically based hydrological model of MCRB (Fang et al., 2013), which was used to assess the impact of forest disturbances on basin hydrology (Pomeroy et al., 2012), analyse antecedent conditions on flood generation (Fang and Pomeroy, 2016), and diagnose rain-on-snow runoff generation for an alpine environment during the 2013 flood in MCRB (Pomeroy et al., 2016).

This paper includes datasets of meteorological, snow survey, streamflow, and groundwater observations measured in MCRB. Meteorological datasets include historical observations by the CFS and ECCC and recent measurements by the University of Saskatchewan Centre for Hydrology. Continuous records of streamflow measurements by ECCC and the University of Saskatchewan as well as groundwater levels monitored by AEP are also included. The snow survey data presented were conducted in clearings, under forest canopies, and on hillslopes at various elevations and are useful for model evaluation and snow process studies. Some of the studies utilising these datasets document the basin resilience to changes in climate, extreme weather, and land cover (Harder et al., 2015), a sensitivity analysis of climate warming on snow processes (Pomeroy et al., 2015), and assessments of variability of climate and its impact on the hydrological processes (Siemens, 2016).

\section{Site description}

Marmot Creek Research Basin (MCRB) $\left(50.95^{\circ} \mathrm{N}\right.$, $115.15^{\circ} \mathrm{W}$ ) is in the headwaters of the Bow River basin in the Front Ranges of the Canadian Rocky Mountains (Fig. 1) and its streamflow discharges into the Kananaskis River. The basin area $\left(9.4 \mathrm{~km}^{2}\right)$ is defined by the Water Survey of Canada stream gauge that was installed in 1962 (Bruce and Clark, 1965). MCRB is composed of three upper sub-basins: Cabin Creek $\left(2.35 \mathrm{~km}^{2}\right)$, Middle Creek $\left(2.94 \mathrm{~km}^{2}\right)$, and Twin Creek $\left(2.79 \mathrm{~km}^{2}\right)$, which converge into the confluence sub-basin above the main stream gauge $\left(1.32 \mathrm{~km}^{2}\right)$. Upper Marmot Creek is an upper sub-basin of Middle Creek $\left(1.178 \mathrm{~km}^{2}\right)$, is primarily alpine, and is also gauged. Based on a resampled 2007 lidar $8 \mathrm{~m}$ digital elevation model (DEM) (Hopkinson et al., 2012), hypsometric curves were derived for MCRB and its three sub-basins (Fig. 2). Elevation ranges from $1590 \mathrm{~m}$ a.s.l. (above sea level) at the main 


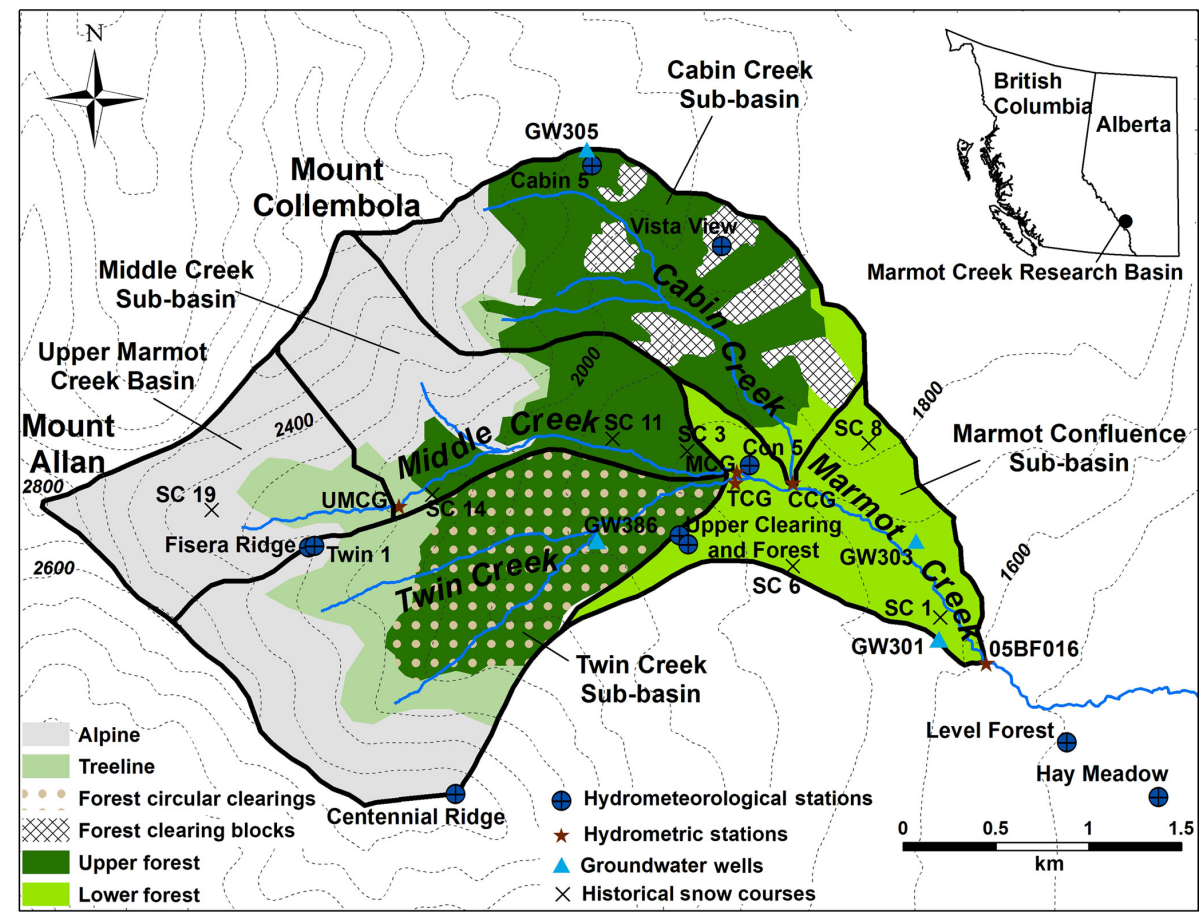

Figure 1. Location and contour map of the Marmot Creek Research Basin (MCRB), showing hydrometeorological stations, hydrometric stations, groundwater wells and snow courses, and ecozones of the MCRB: alpine, treeline, upper forest, forest clearing blocks, forest circular clearings, and lower forest. Note that the size and areas of circular clearings in Twin Creek are not to scale.

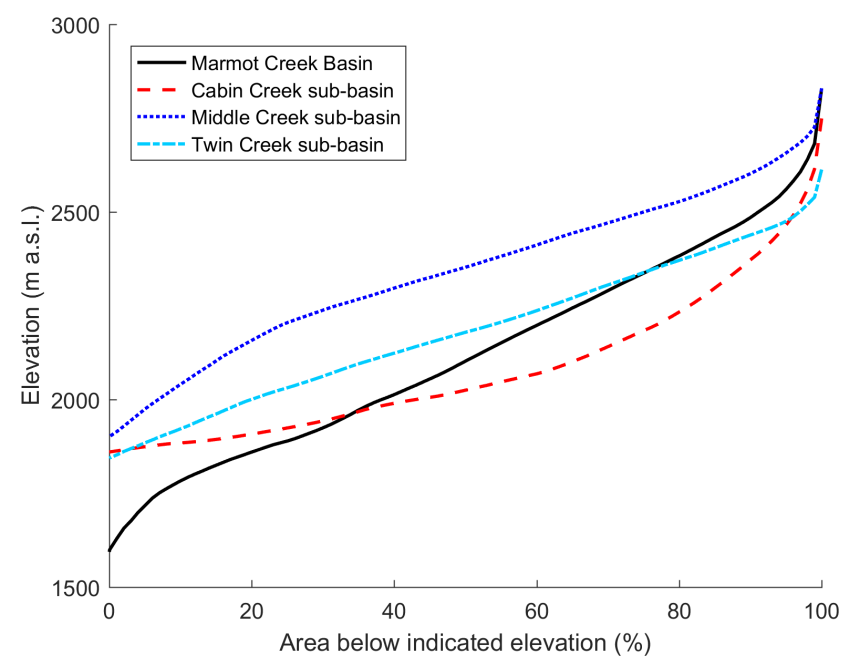

Figure 2. Hypsometric curves for the Marmot Creek Research Basin and three sub-basins showing the relationship between the elevation and percent area below the indicated elevation.

Marmot Creek gauging station to $2829 \mathrm{~m}$ at the summit of Mount Allan. The $8 \mathrm{~m}$ resampled lidar DEM, sub-basin stream network, and sub-basin boundary GIS data are also included in the datasets.

Most of MCRB is covered by needleleaf vegetation, which is dominated by Engelmann spruce (Picea engelmannii) and subalpine fir (Abies lasiocarpa) in upper-mid elevations of the basin (1710 to $2277 \mathrm{~m}$ ). The lower-elevation (1590 to $2015 \mathrm{~m}$ ) forests are mainly Engelmann spruce and lodgepole pine (Pinus contorta var. Latifolia) with trembling aspen (Populus tremuloides) present near the basin outlet (Kirby and Ogilvy, 1969). Alpine larch (Larix lyallii) and short shrubs are present around the treeline at approximately 2016 to $2379 \mathrm{~m}$. Exposed rock surfaces, grasses, and talus are present in the highest alpine part of the basin (1956 to $2829 \mathrm{~m}$ ). Physiographic descriptions of these ecozones are shown in Table 1 and they are mapped in Fig. 1. These ecozones were determined from the forest cover map by the Alberta Forest Service (1963) with recent updates from site visits. Forest management experiments conducted in the 1970s and 1980s left six large clear-cut blocks (1838 to $2062 \mathrm{~m}$ ) in the Cabin Creek sub-basin and numerous small circular forest clearings (1762 to $2209 \mathrm{~m}$ ) in the Twin Creek subbasin (Golding and Swanson, 1986). These old forest clearcut blocks and clearings have regrown as sparse juvenile forest to varying degrees. The basin surface land cover GIS data are included in the datasets.

The surficial soils are primarily poorly developed mountain soils consisting of glaciofluvial, surficial till, and postglacial colluvium deposits (Beke, 1969). Relatively impermeable bedrock is found at the higher elevations, whilst the rest of the basin is covered by a deep layer of coarse and permeable soil allowing for rapid rainfall infiltration to subsur- 
Table 1. Area and mean elevation, aspect, and slope for ecozones at the Marmot Creek Research Basin. Note that the aspect is in degrees clockwise from north.

\begin{tabular}{lcrrrr}
\hline Ecozone & $\begin{array}{r}\text { Area } \\
\left(\mathrm{km}^{2}\right)\end{array}$ & $\begin{array}{r}\text { Area fraction } \\
(\% \text { of basin })\end{array}$ & $\begin{array}{r}\text { Elevation } \\
(\mathrm{m} \text { a.s.1. })\end{array}$ & $\begin{array}{r}\text { Aspect } \\
\left({ }^{\circ}\right)\end{array}$ & $\begin{array}{r}\text { Slope } \\
\left({ }^{\circ}\right)\end{array}$ \\
\hline Alpine & 3.23 & 34.5 & 2413 & 110 & 30 \\
Treeline & 0.93 & 10.0 & 2217 & 91 & 22 \\
Upper forest & 2.75 & 29.3 & 1983 & 108 & 20 \\
Forest clearing blocks & 0.40 & 4.3 & 1927 & 140 & 11 \\
Forest circular clearing north-facing & 0.26 & 2.7 & 1966 & 34 & 17 \\
Forest circular clearing south-facing & 0.24 & 2.6 & 2014 & 113 & 21 \\
Lower forest & 1.42 & 15.2 & 1756 & 113 & 14 \\
\hline
\end{tabular}

face layers overlying relatively impermeable shale (Jeffrey, 1965).

Continental air masses control the weather in the region, which has long and cold winters and cool and wet springs with a late spring-early summer precipitation maximum. Westerly warm and dry Chinook (foehn) winds lead to brief periods when the air temperature exceeds $0{ }^{\circ} \mathrm{C}$ during the winter months - these events can result in snowpack ablation at lower elevations. Annual precipitation ranges from $600 \mathrm{~mm}$ at lower elevations to more than $1100 \mathrm{~mm}$ at the higher elevations, of which approximately $70 \%$ to $75 \%$ occurs as snowfall with the percentage increasing with elevation (Storr, 1967). Mean monthly air temperature ranges from $14^{\circ} \mathrm{C}$ observed at $1850 \mathrm{~m}$ in July to $-10^{\circ} \mathrm{C}$ observed at $2450 \mathrm{~m}$ in January. Mean air temperatures have increased by $2.3^{\circ} \mathrm{C}$ from 1967 to 2013 , but there are no trends in precipitation or streamflow (Harder et al., 2015).

\section{Meteorological data}

\subsection{Recent quality-controlled data}

Quality-controlled (QC) 15 min interval hydrometeorological data were processed from raw data measured at the recent stations in MCRB: Hay Meadow, Level Forest, Upper Clearing, Upper Clearing Tower, Upper Forest, Vista View, Fisera Ridge, and Centennial Ridge. Photos of these stations are shown in Fig. 3, and Table 2 shows a list of the variables in the QC data along with instrumentation, record length, and location for the stations. Most current stations started measurements in 2005 and cover 11 water years (WY) from 1 October 2005 to 30 September 2016 (WY2006 to WY2016) with two exceptions: Upper Clearing Tower and Fisera Ridge; the former started data collection on 21 October 2007 and the latter started data collection on 13 October 2006. The QC data were generated by applying a quality assurance procedure to remove erroneous data in the $15 \mathrm{~min}$ raw data. Table 3 lists the QC thresholds used to remove (1) measurements outside of defined maximum and minimum ranges, (2) measurements that exceed a rate of change (ROC) limit, and (3) constant measurements due to sensor failure. In the QC data, values of -9999 denote the measurements removed from the raw data. In addition, daily QC soil moisture is provided for 11 water years from the Level Forest station and 8 water years (WY2006 to WY2013) from Upper Forest. From 19 October 2012, soil moisture is monitored at a $15 \mathrm{~min}$ interval at Upper Forest and this higher-temporalresolution data are included.

\subsection{Recent modelling data}

Hourly modelling data were obtained by averaging the 15 min QC observations of air temperature $\left({ }^{\circ} \mathrm{C}\right)$, relative humidity $(\%)$, wind speed $\left(\mathrm{m} \mathrm{s}^{-1}\right)$, incoming solar radiation $\left(\mathrm{W} \mathrm{m}^{-2}\right)$, and soil temperature at either 5 or $10 \mathrm{~cm}$ below ground surface $\left({ }^{\circ} \mathrm{C}\right)$ and by summing the $15 \mathrm{~min} \mathrm{QC}$ observation of precipitation (mm). Missing observations of air temperature, relative humidity, wind speed, incoming solar radiation, and soil temperature were filled using either temporal averaging interpolation or linear regression to nearby stations. When intervals of missing data were less than $3 \mathrm{~h}$, temporal averaging was employed where the observations of the variable $3 \mathrm{~h}$ before and $3 \mathrm{~h}$ after the missing interval from the same station were used to calculate the average. When the missing data interval was longer than $3 \mathrm{~h}$, linear regressions were developed amongst stations using the raw data, the regressions were ranked based on $r^{2}$ value, and the regression relationship with the highest $r^{2}$ value was selected to fill in the missing data. For missing precipitation, observations from a nearby station were used along with seasonal precipitation adjustments for elevation to fill in the missing precipitation. The hourly modelling data are provided for 11 water years from 1 October 2005 to 30 September 2016. As described in the previous section, both Fisera Ridge and Upper Clearing Tower stations were established after WY2006, and the hourly modelling data before station establishment were estimated. For the Fisera Ridge station, air temperature, relative humidity, wind speed, incoming solar radiation, and soil temperature from 1 October 2005 to 13 October 2006 were estimated based on linear interpolation to nearby stations, and precipitation from 1 October 2005 to 16 September 2008 was estimated from Upper Clearing precipitation 

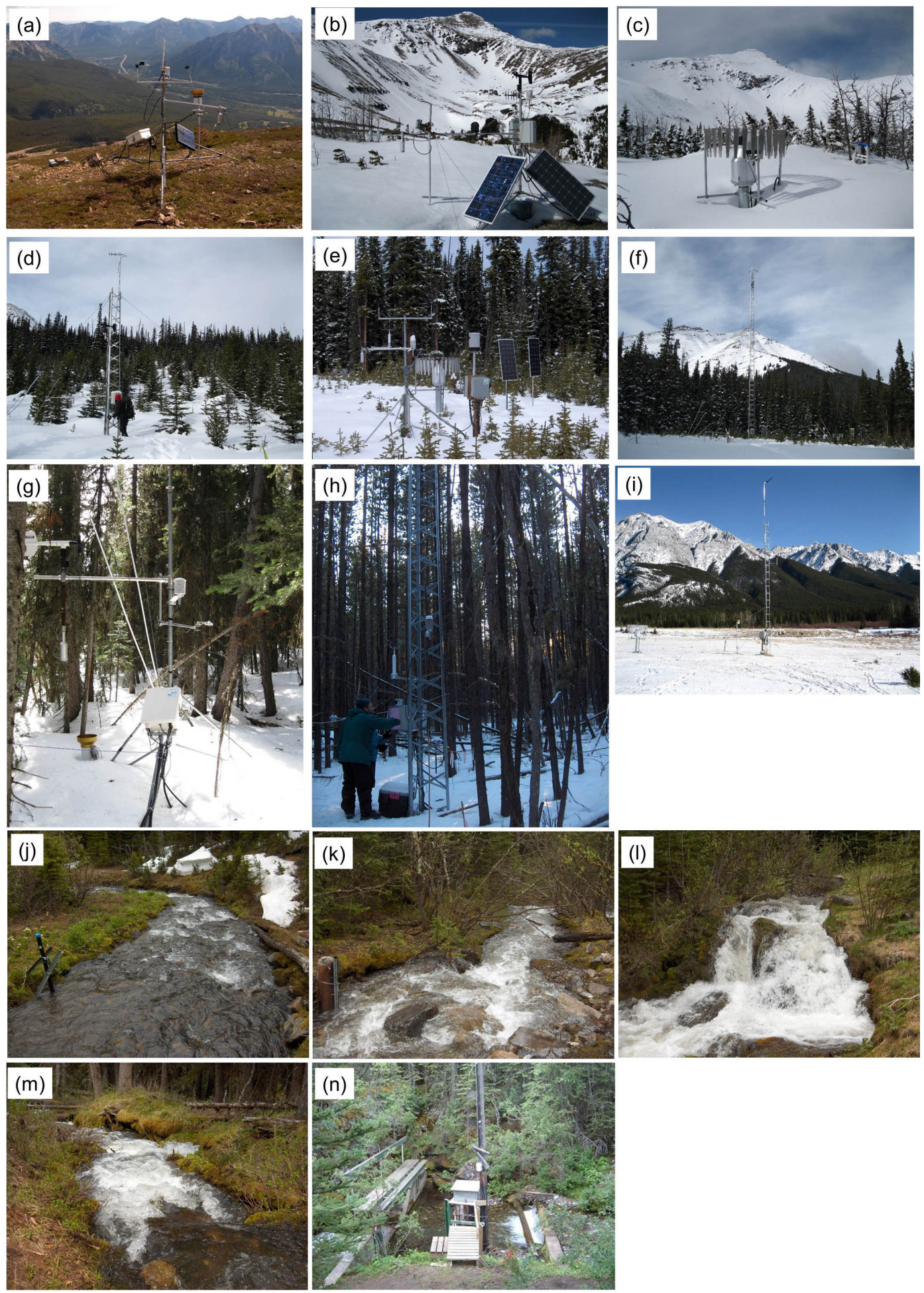

Figure 3. Photos of Marmot Creek Research Basin hydrometeorological and hydrometric stations: (a) Centennial Ridge in July 2010 (2470 m), (b) Fisera Ridge tripod station in April 2015 (2325 m), (c) Fisera Ridge Geonor gauge in March 2011 (2325 m), (d) Vista View in February 2011 (1956 m), (e) Upper Clearing tripod station in May 2010 (1845 m), (f) Upper Clearing Tower station in February 2011 (1845 m), (g) Upper Forest in April 2013 (1848 m), (h) Level Forest in January 2010 (1492 m), (i) Hay Meadow in February 2012 (1436 m), (j) Upper Marmot Creek stream gauge in July 2010 (2200 m), (k) Cabin Creek stream gauge in June 2010 (1710 m), (l) Middle Creek stream gauge in June 2010 (1754 m), (m) Twin Creek stream gauge in June 2010 (1754 m), and (n) Marmot Creek stream gauge in June 2010 (1592 m). 
Table 2. Hydrometeorological variables, instrumentation, and height from the recent stations at the Marmot Creek Research Basin. AGS and BGS denote the distance above ground surface and below ground surface, respectively; n/a denotes not applicable.

\begin{tabular}{|c|c|c|c|c|c|c|c|c|}
\hline Station & Hay Meadow & Level Forest & $\begin{array}{l}\text { Upper } \\
\text { Clearing }\end{array}$ & $\begin{array}{l}\text { Upper Clearing } \\
\text { Tower }\end{array}$ & Upper Forest & Vista View & Fisera Ridge & $\begin{array}{l}\text { Centennial } \\
\text { Ridge }\end{array}$ \\
\hline Coordinates & $\begin{array}{l}50.9441^{\circ} \mathrm{N} ; \\
115.1389^{\circ} \mathrm{W} \\
1436 \mathrm{~m}\end{array}$ & $\begin{array}{l}50.9466^{\circ} \mathrm{N} \\
115.1464^{\circ} \mathrm{W} \\
1492 \mathrm{~m}\end{array}$ & $\begin{array}{l}50.9565^{\circ} \mathrm{N} \\
115.1754^{\circ} \mathrm{W} \\
1845 \mathrm{~m}\end{array}$ & $\begin{array}{l}50.9565^{\circ} \mathrm{N} \\
115.1754^{\circ} \mathrm{W} \\
1845 \mathrm{~m}\end{array}$ & $\begin{array}{l}50.9569^{\circ} \mathrm{N} ; \\
115.1762^{\circ} \mathrm{W} \\
1848 \mathrm{~m}\end{array}$ & $\begin{array}{l}50.9712^{\circ} \mathrm{N} ; \\
115.1722^{\circ} \mathrm{W}, \\
1956 \mathrm{~m}\end{array}$ & $\begin{array}{l}50.9560^{\circ} \mathrm{N} ; \\
115.2041^{\circ} \mathrm{W} \\
2325 \mathrm{~m}\end{array}$ & $\begin{array}{l}50.9571^{\circ} \mathrm{N} ; \\
115.1930^{\circ} \mathrm{W}, \\
2470 \mathrm{~m}\end{array}$ \\
\hline Record & $\begin{array}{l}1 \text { Oct } 2005- \\
30 \text { Sep } 2016\end{array}$ & $\begin{array}{l}10 \text { Mar 2005- } \\
30 \text { Sep } 2016\end{array}$ & $\begin{array}{l}7 \text { Jun 2005- } \\
30 \text { Sep } 2016\end{array}$ & $\begin{array}{l}21 \text { Oct } 2007- \\
30 \text { Sep } 2016\end{array}$ & $\begin{array}{l}7 \text { Jun } 2005- \\
30 \text { Sep } 2016\end{array}$ & $\begin{array}{l}1 \text { Sep 2005- } \\
30 \text { Sep } 2016\end{array}$ & $\begin{array}{l}13 \text { Oct } 2006- \\
30 \text { Sep } 2016\end{array}$ & $\begin{array}{l}24 \text { Jul 2005- } \\
30 \text { Sep } 2016\end{array}$ \\
\hline $\begin{array}{l}\text { Air temperature } \\
\left({ }^{\circ} \mathrm{C}\right) \text { and } \\
\text { relative humidity } \\
(\%) \text { AGS }(\mathrm{m})\end{array}$ & $\begin{array}{l}\text { Vaisala } \\
\text { HMP45C212 } \\
1.86\end{array}$ & $\begin{array}{l}\text { Vaisala } \\
\text { HMP45C212 }\end{array}$ & $\begin{array}{l}\text { Vaisala } \\
\text { HC2-S3 }\end{array}$ & $\begin{array}{l}\text { Vaisala } \\
\text { HMP45C212 }\end{array}$ & $\begin{array}{l}\text { Vaisala } \\
\text { HMP45C212 }\end{array}$ & $\begin{array}{l}\text { Vaisala } \\
\text { HMP45C212 }\end{array}$ & $\begin{array}{l}\text { Vaisala } \\
\text { HMP45C212 }\end{array}$ & $\begin{array}{l}\text { Vaisala } \\
\text { HMP45C212 } \\
1.93\end{array}$ \\
\hline $\begin{array}{l}\text { Wind speed } \\
\left(\mathrm{m} \mathrm{s}^{-1}\right) \text { and } \\
\text { wind direction }\left(^{\circ}\right)\end{array}$ & $\begin{array}{l}\text { RM Young } \\
05305-10 \\
\text { wind } \\
\text { monitor }\end{array}$ & $\begin{array}{l}\text { Met One } 50.5 \\
\text { sonic } \\
\text { anemometer }\end{array}$ & $\begin{array}{l}\text { RM Young } \\
05305-10 \\
\text { wind } \\
\text { monitor }\end{array}$ & $\begin{array}{l}\text { RM Young } \\
05305-10 \\
\text { wind } \\
\text { monitor }\end{array}$ & $\begin{array}{l}\text { RM Young } \\
05305-10 \\
\text { wind } \\
\text { monitor }\end{array}$ & $\begin{array}{l}\text { RM Young } \\
05105-10 \\
\text { wind } \\
\text { monitor }\end{array}$ & $\begin{array}{l}\text { Wind speed and } \\
\text { direction A - } \\
\text { RM Young } \\
05305-10 \\
\text { wind monitor } \\
\text { wind speed B - } \\
\text { three-cup anemometer }\end{array}$ & $\begin{array}{l}\text { RM Young } \\
05105-10 \\
\text { wind } \\
\text { monitor }\end{array}$ \\
\hline AGS (m) & 7 & 2.45 & 2.85 & 18 & 2.77 & 4.11 & $\mathrm{~A}-2.55$ & 2.41 \\
\hline $\begin{array}{l}\text { Snow depth (m) } \\
\text { AGS }(m)\end{array}$ & $\begin{array}{l}\text { SR50 } \\
1.65\end{array}$ & $\begin{array}{l}\text { SR50 } \\
1.04\end{array}$ & $\begin{array}{l}\text { SR50 } \\
1.76\end{array}$ & $\mathrm{n} / \mathrm{a}$ & $\begin{array}{l}\text { SR50 } \\
1.63\end{array}$ & $\begin{array}{l}\text { SR50 } \\
1.59\end{array}$ & $\begin{array}{l}\text { SR50 } \\
1.19\end{array}$ & $\begin{array}{l}\text { SR50 } \\
1.03\end{array}$ \\
\hline $\begin{array}{l}\text { Soil temperature } \\
\left({ }^{\circ} \mathrm{C}\right) \\
\text { BGS }(\mathrm{cm})\end{array}$ & $\begin{array}{l}\text { K-type } \\
\text { thermocouple } \\
\text { A }-5 \\
B-10 \\
C-20\end{array}$ & $\begin{array}{l}\text { K-type } \\
\text { thermocouple } \\
\text { A }-5 \\
B-25 \\
C-40\end{array}$ & $\begin{array}{l}\text { K-type } \\
\text { thermocouple } \\
\text { A }-10 \\
B-20\end{array}$ & $\mathrm{n} / \mathrm{a}$ & $\begin{array}{l}\text { K-type } \\
\text { thermocouple } \\
A-10 \\
B-20\end{array}$ & $\begin{array}{l}\text { K-type } \\
\text { thermocouple } \\
\text { A }-5 \\
\text { B }-10 \\
\text { C }-20\end{array}$ & $\begin{array}{l}\text { CS 107B } \\
\text { thermistor } \\
A-5 \\
B-15\end{array}$ & $\begin{array}{l}\text { CS 107B } \\
\text { thermistor } \\
\mathrm{A}-5 \\
\mathrm{~B}-15\end{array}$ \\
\hline $\begin{array}{l}\text { Soil heat } \\
\text { Flux }\left(\mathrm{W} \mathrm{m}^{-2}\right) \\
\text { BGS }(\mathrm{cm})\end{array}$ & $\begin{array}{l}\text { HFT3 } \\
\text { heat flux } \\
\text { plate } \\
10\end{array}$ & $\begin{array}{l}\text { HFT3 } \\
\text { heat flux } \\
\text { plate } \\
10\end{array}$ & $\begin{array}{l}\text { HFP01 } \\
\text { heat flux } \\
\text { plate } \\
10\end{array}$ & $\mathrm{n} / \mathrm{a}$ & $\mathrm{n} / \mathrm{a}$ & $\begin{array}{l}\text { HFP01 } \\
\text { heat flux } \\
\text { plate } \\
2\end{array}$ & $\begin{array}{l}\text { HFT3 } \\
\text { heat flux } \\
\text { plate } \\
10\end{array}$ & $\mathrm{n} / \mathrm{a}$ \\
\hline $\begin{array}{l}\text { Soil moisture } \\
\left(\mathrm{m}^{3} \mathrm{~m}^{-3}\right) \\
\text { BGS }(\mathrm{cm})\end{array}$ & $\begin{array}{l}\text { CS616 soil } \\
\text { moisture probe } \\
15\end{array}$ & $\begin{array}{l}\text { CS616 soil } \\
\text { moisture probe } \\
25\end{array}$ & $\mathrm{n} / \mathrm{a}$ & $\mathrm{n} / \mathrm{a}$ & $\begin{array}{l}\text { CS616 soil } \\
\text { moisture probe } \\
25\end{array}$ & $\mathrm{n} / \mathrm{a}$ & $\mathrm{n} / \mathrm{a}$ & $\mathrm{n} / \mathrm{a}$ \\
\hline $\begin{array}{l}\text { Incoming solar } \\
\text { radiation }\left(\mathrm{W} \mathrm{m}^{-2}\right) \\
\text { AGS }(\mathrm{m}) \\
\text { Outgoing solar } \\
\text { radiation }\left(\mathrm{W} \mathrm{m}^{-2}\right)\end{array}$ & $\begin{array}{l}\text { Kipp and } \\
\text { Zonen } \\
\text { CM3 } \\
\text { pyranometers }\end{array}$ & $\begin{array}{l}\text { Kipp and } \\
\text { Zonen } \\
\text { CM3 } \\
\text { pyranometers }\end{array}$ & $\begin{array}{l}\text { Kipp and } \\
\text { Zonen } \\
\text { CM3 } \\
\text { pyranometers }\end{array}$ & $\begin{array}{l}\text { Kipp and } \\
\text { Zonen CM21 } \\
\text { pyranometer, } \\
20\end{array}$ & $\begin{array}{l}\text { Kipp and } \\
\text { Zonen } \\
\text { CM3 } \\
\text { pyranometers }\end{array}$ & $\begin{array}{l}\text { Apogee } \\
\text { CS300-L } \\
\text { pyranometer, } \\
1.97\end{array}$ & $\begin{array}{l}\text { Kipp and } \\
\text { Zonen } \\
\text { CM3 } \\
\text { pyranometers }\end{array}$ & $\begin{array}{l}\mathrm{Li} \text {-cor LI200s } \\
\text { shortwave } \\
\text { radiometer }\end{array}$ \\
\hline $\begin{array}{l}\text { AGS }(\mathrm{m}) \\
\text { Incoming longwave } \\
\text { radiation }\left(\mathrm{W} \mathrm{m}^{-2}\right)\end{array}$ & $\begin{array}{l}1.95 \\
\text { Kipp and Zonen }\end{array}$ & $\begin{array}{l}1.31 \\
\text { Kipp and Zonen }\end{array}$ & $\begin{array}{l}2.33 \\
\text { Kipp and Zonen }\end{array}$ & $\begin{array}{l}\text { n/a } \\
\text { Kipp and Zonen } \\
\text { CG1 }\end{array}$ & $\begin{array}{l}1.95 \\
\text { Kipp and Zonen }\end{array}$ & $\begin{array}{l}\mathrm{n} / \mathrm{a} \\
\mathrm{n} / \mathrm{a}\end{array}$ & $\begin{array}{l}1.45 \\
\text { Kipp and Zonen }\end{array}$ & 1.37 \\
\hline $\begin{array}{l}\text { AGS }(\mathrm{m}) \\
\text { Outgoing longwave } \\
\text { radiation }\left(\mathrm{W} \mathrm{m}^{-2}\right)\end{array}$ & $\begin{array}{l}\text { CG3 } \\
\text { pyranometers }\end{array}$ & $\begin{array}{l}\text { CG3 } \\
\text { pyranometers }\end{array}$ & $\begin{array}{l}\text { CG3 } \\
\text { pyranometers }\end{array}$ & $\begin{array}{l}\text { pyrgeometer, } \\
20\end{array}$ & $\begin{array}{l}\text { CG3 } \\
\text { pyranometers }\end{array}$ & 1.97 & $\begin{array}{l}\text { CG3 } \\
\text { pyranometers }\end{array}$ & $\mathrm{n} / \mathrm{a}$ \\
\hline $\operatorname{AGS}(\mathrm{m})$ & 1.95 & 1.31 & 2.33 & $\mathrm{n} / \mathrm{a}$ & 1.95 & $\mathrm{n} / \mathrm{a}$ & 1.45 & $\mathrm{n} / \mathrm{a}$ \\
\hline $\operatorname{AGS}(\mathrm{m})$ & $\begin{array}{l}\text { Texas } \\
\text { TE525M } \\
\text { rain } \\
\text { gauge } \\
2.56\end{array}$ & $\mathrm{n} / \mathrm{a}$ & $\begin{array}{l}\text { Hydrological } \\
\text { Services TB4 } \\
\text { tipping-bucket } \\
\text { rain gauge } \\
2.36\end{array}$ & $\mathrm{n} / \mathrm{a}$ & $\begin{array}{l}\text { Texas } \\
\text { TE525M } \\
\text { rain } \\
\text { gauge } \\
0.7\end{array}$ & $\mathrm{n} / \mathrm{a}$ & $\begin{array}{l}\text { Hydrological } \\
\text { Services TB4 } \\
\text { tipping-bucket } \\
\text { rain gauge } \\
4.2\end{array}$ & $\begin{array}{l}\text { Texas } \\
\text { TE525M } \\
\text { rain } \\
\text { gauge } \\
1.56\end{array}$ \\
\hline $\begin{array}{l}\text { All precipitation } \\
(\mathrm{mm}) \\
\text { AGS }(\mathrm{m})\end{array}$ & $\begin{array}{l}\text { Geonor T200B } \\
\text { gauge with } \\
\text { alter shield } \\
1.8\end{array}$ & $\mathrm{n} / \mathrm{a}$ & $\begin{array}{l}\text { Geonor T200B } \\
\text { gauge with } \\
\text { alter shield } \\
1.85\end{array}$ & $\mathrm{n} / \mathrm{a}$ & $\mathrm{n} / \mathrm{a}$ & $\mathrm{n} / \mathrm{a}$ & $\begin{array}{l}\text { Geonor T200B } \\
\text { gauge with } \\
\text { alter shield } \\
4.1\end{array}$ & $\mathrm{n} / \mathrm{a}$ \\
\hline $\begin{array}{l}\text { Barometric } \\
\text { pressure } \\
(\mathrm{mb})\end{array}$ & $\begin{array}{l}\text { BP61025V } \\
\text { pressure } \\
\text { sensor }\end{array}$ & $\mathrm{n} / \mathrm{a}$ & $\begin{array}{l}\text { CS106 } \\
\text { barometric } \\
\text { pressure } \\
\text { sensor } \\
1.25\end{array}$ & $\mathrm{n} / \mathrm{a}$ & $\mathrm{n} / \mathrm{a}$ & $\mathrm{n} / \mathrm{a}$ & $\mathrm{n} / \mathrm{a}$ & $\begin{array}{l}\text { BP61025V } \\
\text { pressure } \\
\text { sensor } \\
0.7\end{array}$ \\
\hline
\end{tabular}


Table 3. Quality-controlled threshold values for $15 \mathrm{~min}$ hydrometeorological variables for current stations in MCRB; ROC and n/a denote rate of change and not applicable, respectively.

\begin{tabular}{llrrrr}
\hline Variable & Unit & Maximum & Minimum & ROC limit & $\begin{array}{r}\text { Time steps to flag } \\
\text { constant value }\end{array}$ \\
\hline Air temperature & ${ }^{\circ} \mathrm{C}$ & 40 & -60 & 10 & 16 \\
Relative humidity & $\%$ & 100 & 0 & $30 \%$ & 16 \\
Wind speed & $\mathrm{m} \mathrm{s}^{-1}$ & 30 & 0 & $\mathrm{n} / \mathrm{a}$ & 16 \\
Snow depth & $\mathrm{m}$ & 5 & 0 & $\mathrm{n} / \mathrm{a}$ & $\mathrm{n} / \mathrm{a}$ \\
Soil temperature & ${ }^{\circ} \mathrm{C}$ & 50 & -40 & 10 & 96 \\
Soil heat flux & $\mathrm{W} \mathrm{m}^{-2}$ & 1000 & -500 & 100 & 16 \\
Soil moisture & $\mathrm{fraction}_{\text {Solar radiation }}$ & 1 & 0 & 0.2 & 16 \\
Longwave radiation & $\mathrm{W} \mathrm{m}^{-2}$ & 1368 & 0 & 1450 & 48 \\
Precipitation & 600 & 100 & 300 & 16 \\
Barometric pressure & $\mathrm{mb}$ & 30 & 0 & $\mathrm{n} / \mathrm{a}$ & $\mathrm{n} / \mathrm{a}$ \\
\hline
\end{tabular}

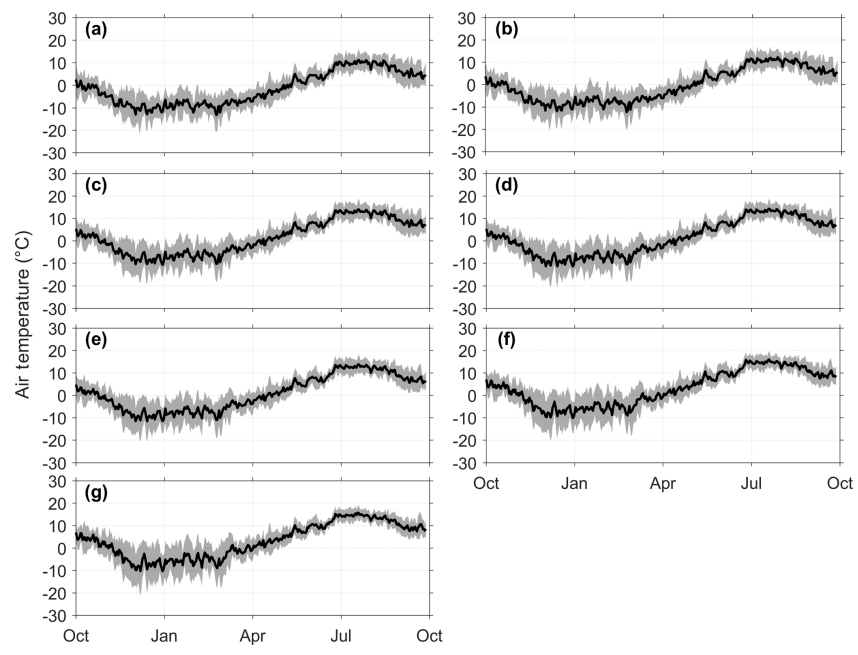

Figure 4. Annual mean daily air temperature for 11 water years from 1 October 2005 to 30 September 2016 at MCRB stations: (a) Centennial Ridge, (b) Fisera Ridge, (c) Vista View, (d) Upper Clearing, (e) Upper Forest, (f) Level Forest, and (g) Hay Meadow. The line represents the annual mean and the shaded area represents the standard deviation of the 11-year daily air temperature.

with seasonal precipitation adjustments for elevation. For the Upper Clearing Tower station, the hourly incoming solar radiation measured at $20 \mathrm{~m}$ above ground is provided, and from 1 October 2005 to 21 October 2007 it was estimated from incoming solar radiation measured at the lower-level Upper Clearing tripod station based on a linear regression because of the location of both stations in the same forest clearing. Figures 4-8 show the annual mean daily air temperature, relative humidity, wind speed, incoming solar radiation, and accumulated rainfall and snowfall with their inter-annual variability for MCRB stations for the 11 water years.

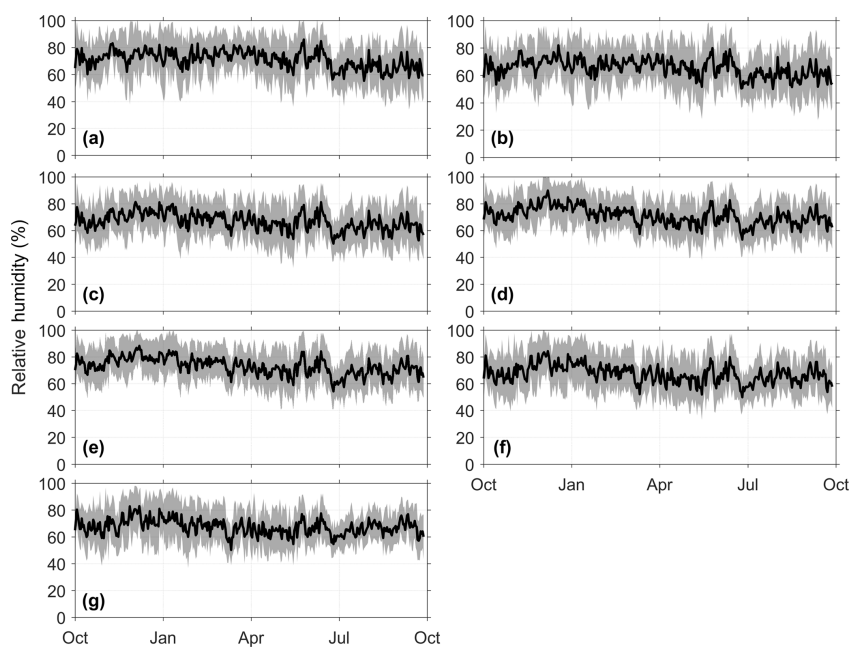

Figure 5. Annual mean daily relative humidity for 11 water years from 1 October 2005 to 30 September 2016 at MCRB stations: (a) Centennial Ridge, (b) Fisera Ridge, (c) Vista View, (d) Upper Clearing, (e) Upper Forest, (f) Level Forest, and (g) Hay Meadow. The line represents the annual mean and the shaded area represents the standard deviation of the 11-year daily relative humidity.

\subsubsection{Air temperature and relative humidity}

Air temperature and relative humidity were measured using Vaisala hygrothermometers with naturally ventilated Gill radiation shields at all seven stations. Table 4 shows that average air temperature at MCRB for the 11 water years ranges from $-1.6^{\circ} \mathrm{C}$ at the Centennial Ridge station to $-0.4{ }^{\circ} \mathrm{C}$ at the Fisera Ridge station. Both stations are located on alpine ridgetops, above treeline. Higher temperatures are found at lower elevations, where the 11-year average air temperature is 1.4 and $3.1{ }^{\circ} \mathrm{C}$ for the Upper Clearing station in a montane forest and the Hay Meadow station on the valley floor, respectively. WY2016 was the warmest, with the average water year air temperature being $-0.3,1.0,2.7$, and $4.4^{\circ} \mathrm{C}$ for 


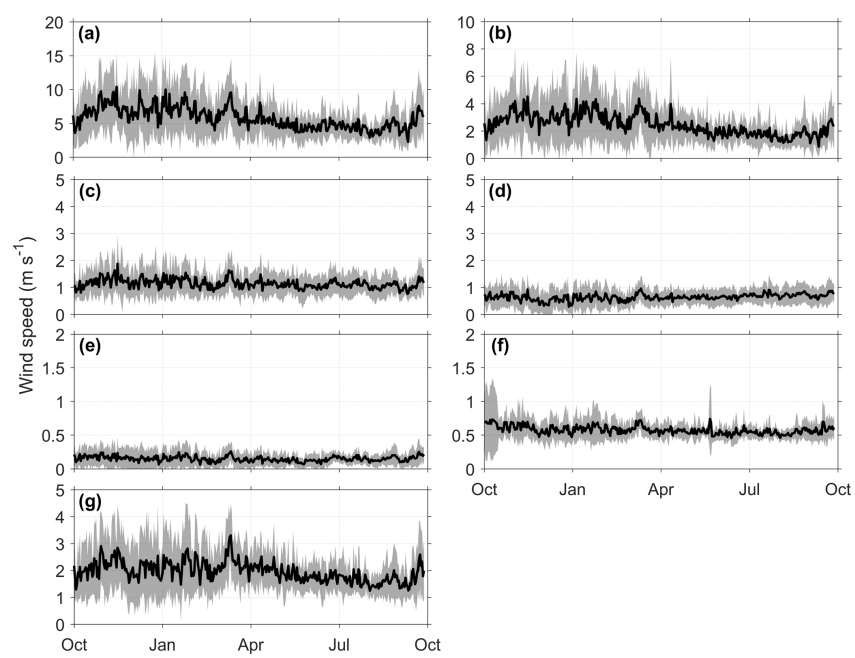

Figure 6. Annual mean daily wind speed for 11 water years from 1 October 2005 to 30 September 2016 at MCRB stations: (a) Centennial Ridge, (b) Fisera Ridge, (c) Vista View, (d) Upper Clearing, (e) Upper Forest, (f) Level Forest, and (g) Hay Meadow. The line represents the annual mean and the shaded area represents the standard deviation of the 11-year daily wind speed.
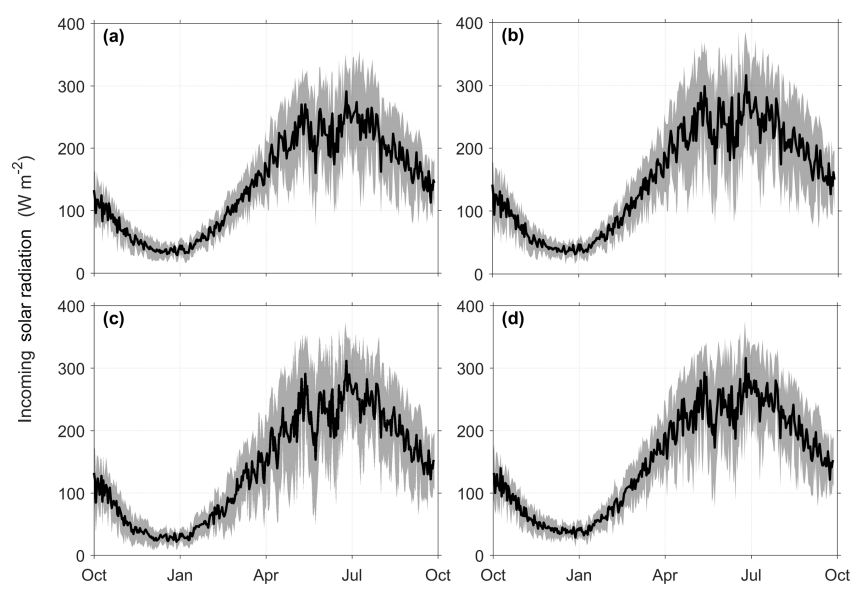

Figure 7. Annual mean daily incoming solar radiation for 11 water years from 1 October 2005 to 30 September 2016 at MCRB stations: (a) Centennial Ridge, (b) Fisera Ridge, (c) Upper Clearing Tower, and (d) Hay Meadow. The line represents the annual mean and the shaded area represents the standard deviation of the 11-year daily incoming solar radiation.

the Centennial Ridge, Fisera Ridge, Upper Clearing, and Hay Meadow stations, respectively. WY2008 was the coolest for the Centennial Ridge and Fisera Ridge stations, with average air temperatures of -2.7 and $-1.7^{\circ} \mathrm{C}$, respectively; whereas WY2011 was the coolest for the Upper Clearing and Hay Meadow stations, with average air temperatures of 0.4 and $1.9^{\circ} \mathrm{C}$ for the Upper Clearing and Hay Meadow stations, respectively. An example of hourly air temperature and rela-

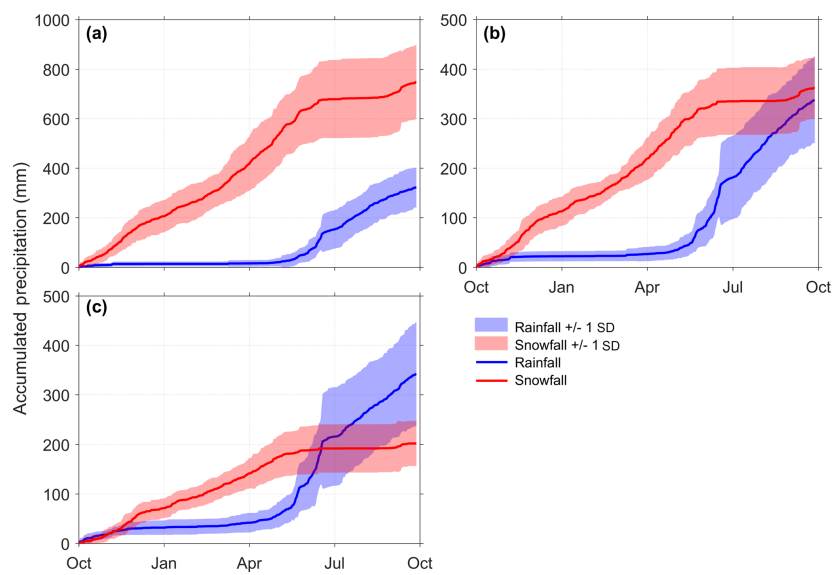

Figure 8. Annual mean daily accumulated rainfall and snowfall for 11 water years from 1 October 2005 to 30 September 2016 at MCRB stations: (a) Fisera Ridge, (b) Upper Clearing, and (c) Hay Meadow. The line represents the annual mean and the shaded area represents the standard deviation of the 11-year daily accumulated rainfall and snowfall. Rainfall and snowfall are calculated from wind-corrected storage-gauge observations with precipitation phase calculated as per Harder and Pomeroy (2013).

tively humidity from Fisera Ridge station is shown in Fig. 9a and $b$.

\subsubsection{Wind speed}

Wind speeds were measured at all seven stations using propeller-type RM Young anemometers. The 11-water-year average wind speeds on wind-exposed alpine ridges are 5.8 and $2.5 \mathrm{~m} \mathrm{~s}^{-1}$ at Centennial Ridge measured $(2.41 \mathrm{~m}$ a.g.s., above ground surface) and Fisera Ridge (2.55 m a.g.s.) stations, respectively. Hay Meadow, located on an open grassland valley floor $(7 \mathrm{~m}$ a.g.s.) has an 11-water-year average wind speed of $2.0 \mathrm{~m} \mathrm{~s}^{-1}$. Vista View station $(4.11 \mathrm{~m}$ a.g.s.) is located in a large forest cut block with a short sparse forest cover of young trees and has an 11-water-year average wind speed of $1.1 \mathrm{~m} \mathrm{~s}^{-1}$. For the wind-sheltered stations (Upper Clearing measured at $2.85 \mathrm{~m}$ a.g.s, Upper Forest measured at $2.77 \mathrm{~m}$ a.g.s, and Level Forest measured at $2.45 \mathrm{~m}$ a.g.s), the 11-water-year average wind speeds range from 0.1 to $0.6 \mathrm{~m} \mathrm{~s}^{-1}$. The maximum hourly wind speed recorded during 11 water years is $37.9 \mathrm{~m} \mathrm{~s}^{-1}$ from the Centennial Ridge station. An example of hourly wind speed from the Fisera Ridge station is shown in Fig. 9c.

\subsubsection{Incoming solar radiation}

Incoming solar radiation was measured at all seven stations using Kipp and Zonen pyranometers and is included in the hourly modelling dataset except for the Vista View station due to short length of measurement. For the Upper Clearing site, hourly incoming solar radiation measured at the top of 
Table 4. Mean water year air temperature and total water year precipitation from the current stations at the Marmot Creek Research Basin. Values inside parentheses are total water year snowfall.

\begin{tabular}{lccccccc|rrr}
\hline Water year & \multicolumn{7}{c|}{ Mean air temperature $\left({ }^{\circ} \mathrm{C}\right)$} & \multicolumn{3}{c}{ Total precipitation $(\mathrm{mm})$} \\
\cline { 2 - 10 } & $\begin{array}{c}\text { Centennial } \\
\text { Ridge }\end{array}$ & $\begin{array}{r}\text { Fisera } \\
\text { Ridge }\end{array}$ & $\begin{array}{c}\text { Vista } \\
\text { View }\end{array}$ & $\begin{array}{c}\text { Upper } \\
\text { Clearing }\end{array}$ & $\begin{array}{c}\text { Upper } \\
\text { Forest }\end{array}$ & $\begin{array}{c}\text { Hay } \\
\text { Meadow }\end{array}$ & $\begin{array}{c}\text { Level } \\
\text { Forest }\end{array}$ & $\begin{array}{r}\text { Fisera } \\
\text { Ridge }\end{array}$ & $\begin{array}{r}\text { Upper } \\
\text { Clearing }\end{array}$ & $\begin{array}{r}\text { Hay } \\
\text { Meadow }\end{array}$ \\
\hline 2006 & -1.2 & 0.1 & 2.9 & 2.3 & 1.7 & 4.0 & 4.0 & $902(551)$ & $646(306)$ & $492(155)$ \\
2007 & -1.7 & -0.5 & 2.2 & 1.5 & 0.8 & 3.3 & 3.4 & $1215(815)$ & $797(421)$ & $631(196)$ \\
2008 & -2.7 & -1.7 & 0.8 & 0.6 & 0.0 & 2.3 & 2.4 & $1218(926)$ & $804(421)$ & $693(231)$ \\
2009 & -1.4 & -0.7 & 1.4 & 1.0 & 0.4 & 2.8 & 2.8 & $944(638)$ & $610(332)$ & $450(210)$ \\
2010 & -2.1 & -1.0 & 0.6 & 0.6 & 0.0 & 2.6 & 2.4 & $1140(904)$ & $670(410)$ & $476(205)$ \\
2011 & -2.4 & -1.2 & 0.4 & 0.4 & -0.2 & 1.9 & 1.8 & $1128(865)$ & $671(396)$ & $522(271)$ \\
2012 & -1.4 & -0.2 & 1.5 & 1.6 & 1.0 & 3.6 & 3.6 & $1247(922)$ & $794(419)$ & $586(201)$ \\
2013 & -1.5 & -0.2 & 1.3 & 1.4 & 1.1 & 3.1 & 3.0 & $1329(794)$ & $868(320)$ & $762(207)$ \\
2014 & -2.1 & -0.8 & 0.7 & 0.5 & 0.2 & 2.2 & 2.1 & $877(658)$ & $650(419)$ & $510(267)$ \\
2015 & -0.4 & 0.9 & 2.3 & 2.5 & 2.2 & 4.2 & 4.2 & $857(543)$ & $593(272)$ & $440(163)$ \\
2016 & -0.3 & 1.0 & 2.4 & 2.7 & 2.4 & 4.4 & 4.5 & $939(614)$ & $591(268)$ & $426(118)$ \\
\hline $11-$ water- & & & & & & & & & & \\
year mean & -1.6 & -0.4 & 1.5 & 1.4 & 0.9 & 3.1 & 3.1 & $1070(748)$ & $699(362)$ & $545(202)$ \\
\hline
\end{tabular}

the $20 \mathrm{~m}$ tower station is provided in addition to that from the main tripod station near the ground $(1.95 \mathrm{~m})$. For the subcanopy measurements at the Upper Forest (i.e. mature spruce forest) and Level Forest (i.e. mature lodgepole forest) stations, the 11-water-year mean values range from $15.9 \mathrm{~W} \mathrm{~m}^{-2}$ (Upper Forest) to $23.7 \mathrm{~W} \mathrm{~m}^{-2}$ (Level Forest). For the stations not affected by forest canopy, the 11-water-year mean value ranges from $140.1 \mathrm{~W} \mathrm{~m}^{-2}$ (Upper Clearing $20 \mathrm{~m}$ tower) to $150.3 \mathrm{~W} \mathrm{~m}^{-2}$ (Fisera Ridge). An example of hourly incoming solar radiation from the Fisera Ridge station is shown in Fig. 9d.

\subsubsection{Soil temperature}

Soil temperature was measured using thermistors at all seven stations at either 5 or $10 \mathrm{~cm}$ below ground surface. The 11-water-year mean value ranges from $-0.7^{\circ} \mathrm{C}$ (Centennial Ridge) to $6.5^{\circ} \mathrm{C}$ (Hay Meadow). The maximum hourly soil temperature during 11 water years was $36.6^{\circ} \mathrm{C}$ at the Hay Meadow station and the minimum hourly soil temperature during 11 water years was $-16.5^{\circ} \mathrm{C}$ at the Centennial Ridge station. An example of hourly soil temperature from the Fisera Ridge station is shown in Fig. 9e.

\subsubsection{Precipitation}

Precipitation was measured with Alter-shielded Geonor T200B weighing precipitation gauges at the Hay Meadow, Upper Clearing, and Fisera Ridge stations, and it was corrected for wind-induced undercatch for the wind-exposed Fisera Ridge and Hay Meadow stations (Smith, 2007). Precipitation is divided into rainfall and snowfall based on the psychrometric energy balance precipitation phase determination method developed by Harder and Pomeroy (2013).
Table 4 shows that the average annual precipitation for the 11 water years is $627 \mathrm{~mm}$ ( $229 \mathrm{~mm}$ snow), $839 \mathrm{~mm}$ ( $443 \mathrm{~mm}$ snow), and $1190 \mathrm{~mm}$ (802 mm snow) for Hay Meadow, Upper Clearing, and Fisera Ridge, respectively. The highest annual precipitation during the 11 water years from the Fisera Ridge station was $1329 \mathrm{~mm}$ in WY2013 when approximately $250 \mathrm{~mm}$ of rainfall and snowfall fell during the June 2013 flood (Pomeroy et al., 2016), which also produced the highest annual rainfall $(535 \mathrm{~mm})$ recorded during the 11 water years. An example of hourly cumulative precipitation, divided into rainfall and snowfall from the Fisera Ridge station, is shown in Fig. 9f.

\subsection{Historical modelling data}

Historical meteorological data are available from the three sites shown in Fig. 1. Observations from Confluence $5\left(\right.$ Con $\left.5,50.960^{\circ} \mathrm{N}, 115.171^{\circ} \mathrm{W}, 1770 \mathrm{~m}\right)$, Cabin 5 $\left(50.975^{\circ} \mathrm{N}, 115.182^{\circ} \mathrm{W}, 2051 \mathrm{~m}\right)$, and Twin $1\left(50.957^{\circ} \mathrm{N}\right.$, $115.204^{\circ} \mathrm{W}, 2285 \mathrm{~m}$ ) are provided. These sites were established in the early 1960s by the CFS and ECCC. Based on the availability of data, continuous records of hourly air temperature $\left({ }^{\circ} \mathrm{C}\right)$, relative humidity $(\%)$, wind speed $\left(\mathrm{m} \mathrm{s}^{-1}\right)$, and daily precipitation $(\mathrm{mm})$ are included for 18 water years from 1 October 1969 to 30 September 1987. Air temperature and relative humidity were measured by thermographs or hygrothermographs (Munn and Storr, 1967); wind speed was measured by an MSC-type 45B anemometer, and for precipitation, Leupold-Stevens Q12M weighing gauges and MSC (Meteorological Service of Canada) tipping-bucket gauges were used to take measurements for snowfall and rainfall, respectively (Storr, 1973). Data quality assurance was undertaken to generate the continuous data from the original obser- 
vations, which includes removing inconsistent measurements and outliers, filling missing data with linear regressions to nearby stations. Details regarding the quality assurance are provided by Siemens (2016). The original measured data are also provided for these sites.

\section{Snow survey data}

\subsection{Recent snow survey data}

Snow survey data collected from transects near the recent meteorological stations Hay Meadow, Level Forest, Upper Clearing, Upper Forest, Vista View, and Fisera Ridge are provided for nine WYs from 2007 to 2016, except for the Hay Meadow in WY 2007 when no measurements were taken. The snow survey data include snow depth, density, and snow water equivalent (SWE). In addition, the snow survey data contain field notes on land cover information of each snow survey transect. The snow surveys usually occur monthly during the winter accumulation period and fortnightly to weekly during the spring melt period. Snow depth was measured by a $1 \mathrm{~m}$ ruler for shallow snowpack or a $3 \mathrm{~m}$ measuring probe for deep snowpacks, and snow density was measured using an ESC30 snow tube for shallow snowpacks or a Mount Rose snow sampler for deeper snowpacks. At each transect, snow depth was observed at $5 \mathrm{~m}$ intervals, and one snow density was collected for every five depth measurements.

\subsection{Historical snow survey data}

Snow survey data collected by CFS from seven snow courses (SCs): 1, 3, 6, 8, 11, 14, and 19 are provided for the water years from 1963 to 1986 . The location of these snow courses is shown in Fig. 1, and a brief description for each snow course is listed in Table 5. Regular measurements were carried out monthly from February to June, and each course consisted of 10 staked points where snow depth and snow water equivalent were measured. In some years, measurements were conducted more than once per month, which provided more details of seasonal snow accumulation. Both monthly snow survey data from 1963 to 1986 and detailed survey data from measurements more than once per month during 1963 to 1980 are included for the historical period. An example of mean transect SWE from historical and recent snow surveys for alpine and montane forest sites is shown in Fig. 10.

\section{Streamflow data}

\subsection{Recent streamflow data}

Recently streamflow observations were made by the University of Saskatchewan starting in spring 2007 at the sub-basin outlets of Cabin, Middle, Twin, and Upper Marmot Creeks and at the basin outlet after the June 2013 flood. Measure- ments at outlets of Cabin, Middle, and Twin Creeks ceased in June 2013 as all three gauging stations (and 2013 data holding data loggers) were destroyed in June 2013. The sites are now difficult to access as the road was destroyed, the channels are unstable, and access trails are covered with fallen trees. Flow depth was continuously measured at 15 min intervals with automated pressure transducers, and velocity was manually measured with a handheld SonTek FlowTracker acoustic Doppler velocimeter every few weeks from spring to autumn. Discharge at $15 \mathrm{~min}$ intervals is calculated based on rating curves from continuous flow depth and frequently manually measured velocity throughout the spring, summer, and autumn. Hourly average streamflow $\left(\mathrm{m}^{3} \mathrm{~s}^{-1}\right)$ is estimated from the $15 \mathrm{~min}$ discharge and is provided for Cabin, Middle, and Twin Creeks from 2007 to 2012, Upper Marmot Creek from 2007 to 2016, and Marmot Creek from 26 June 2013 to 2016.

\subsection{Historical streamflow data}

Daily average streamflow $\left(\mathrm{m}^{3} \mathrm{~s}^{-1}\right)$ was estimated for Cabin Creek, Middle Creek, Twin Creek, and Upper Marmot Creek for the historical period from 1963 to 1986. Streamflow measurements were made by ECCC's Water Survey of Canada at the outlets of the respective sub-basins: Cabin Creek gauge (CCG, 05BF019), Middle Creek gauge (MCG, 05BF017), Twin Creek gauge (TCG, 05BF018), and Upper Marmot Creek gauge (UMCG, 05BF020) shown in Fig. 1. Year-round streamflow discharge was estimated using stage records from flow through V-notch weirs on Middle and Twin Creeks and an H-flume on Cabin and Upper Marmot Creeks (Canadian Forestry Service, 1976; Harder et al., 2015). The Upper Marmot gauge is located higher up the Middle Creek sub-basin and captures the streamflow generated from a predominantly alpine area. The record for Upper Marmot Creek is sporadic due to the ephemeral nature of Middle Creek at this location and site access challenges.

For the Marmot Creek outlet, streamflow was measured by ECCC at the Marmot Creek basin outlet V-notch gauging station (05BF016). The streamflow data span from 1962 to 19 June 2013 and are continuous until 1986 and seasonal thereafter. However, the gauging station was severely damaged in the June 2013 flood (Pomeroy et al., 2016), after which no measurements have been made by ECCC. The University of Saskatchewan restored discharge measurements at this site on 26 June 2013 as described in the previous section. The daily average streamflow data for all sub-basins and Marmot Creek can be searched and then accessed from the ECCC Water Survey of Canada "historical hydrometric data search" website at https://wateroffice.ec.gc.ca/search/ historical_e.html (last access: 1 October 2018). The Water Survey of Canada is preparing to restore this gauge in the near future. 


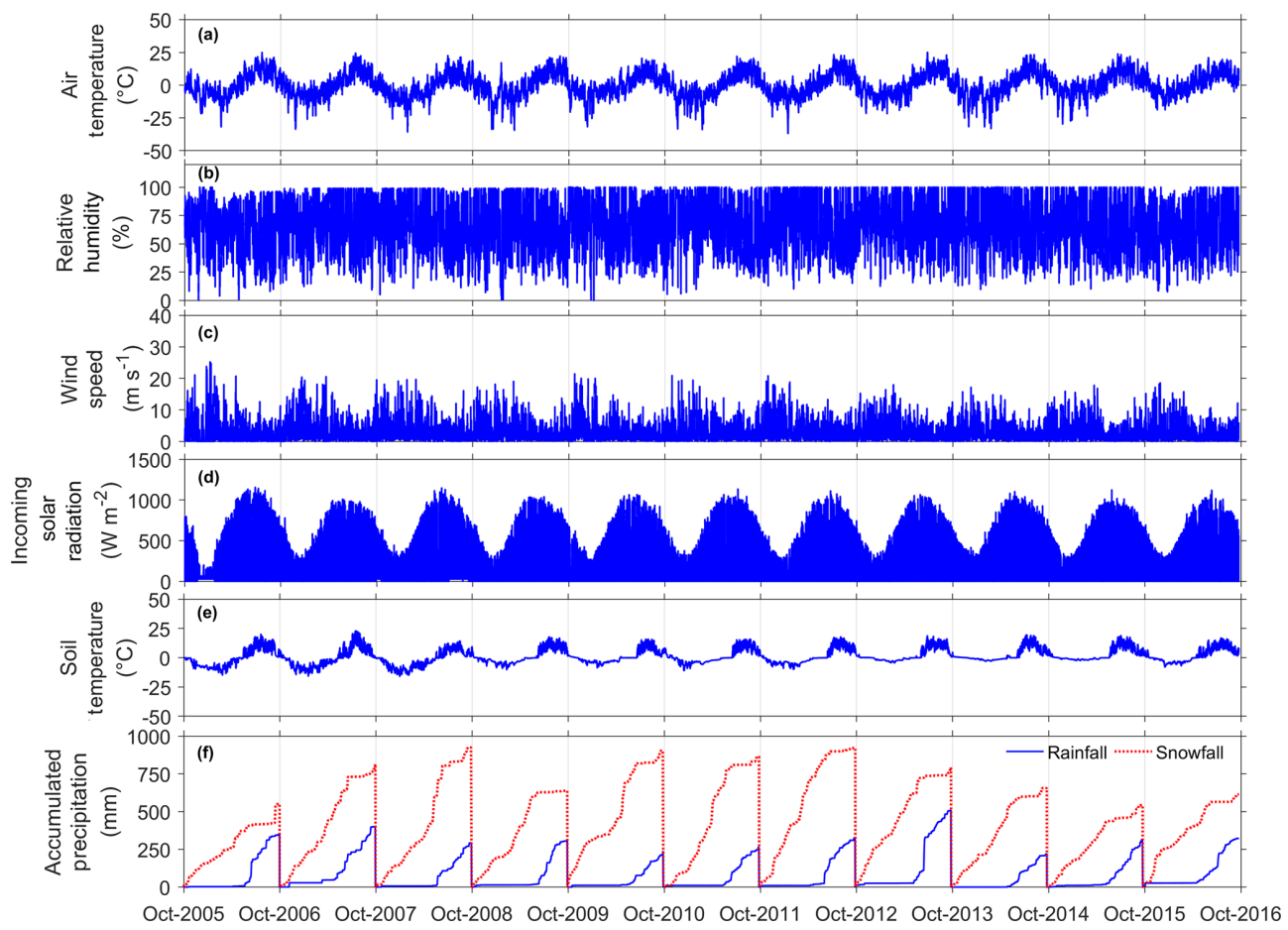

Figure 9. Example of hourly-averaged forcing data from the Fisera Ridge station showing (a) air temperature, (b) relative humidity, (c) wind speed, (d) soil temperature, and (e) rainfall and snowfall for water years starting 1 October. All data are developed from observations except rainfall and snowfall, which are calculated from wind-corrected storage-gauge observations with precipitation phase calculated as per Harder and Pomeroy (2013).

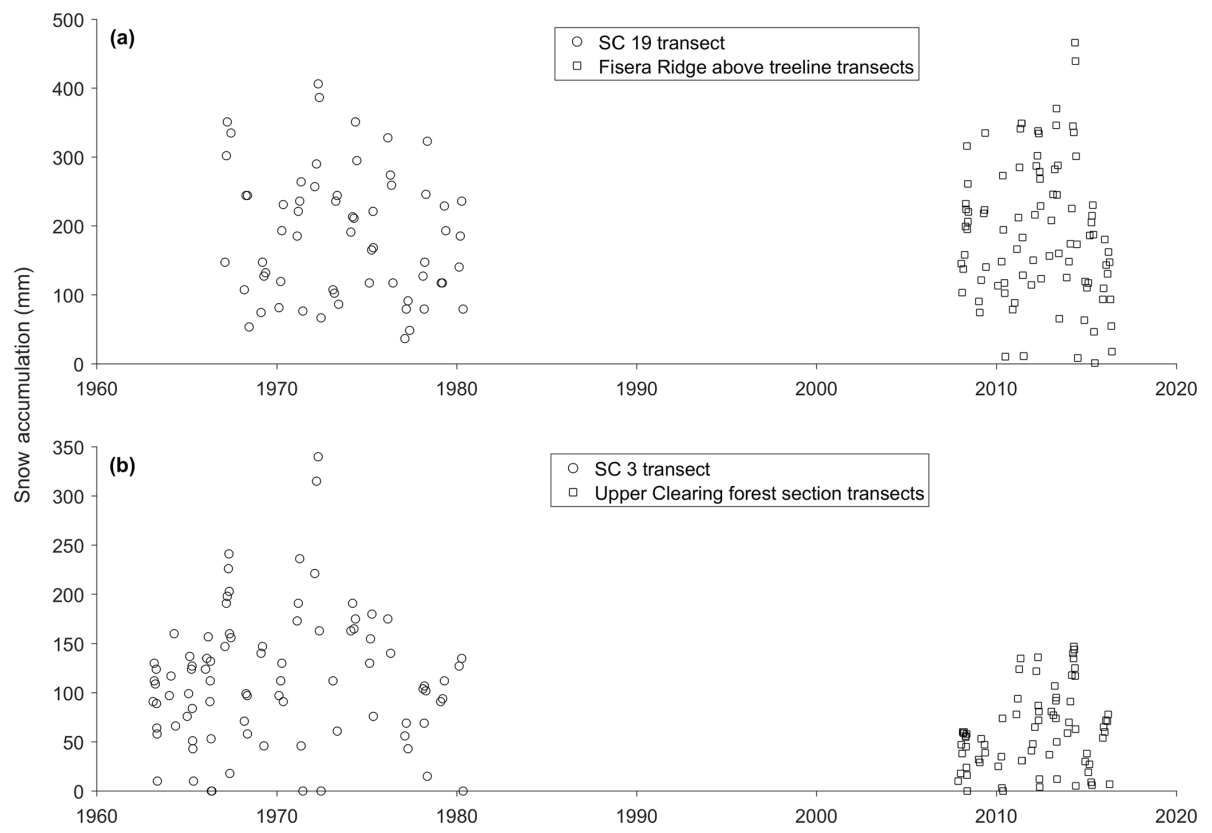

Figure 10. Example of mean transect snow accumulation (SWE) from (a) alpine and (b) montane forest snow survey transects. The historical SWE for alpine and montane forest is from SC 19 and SC 3 transects, respectively. The recent SWE for alpine and montane forest is from Fisera Ridge above treeline transects and Upper Clearing forest section transects, respectively. 
Table 5. Historical snow courses (SCs) at the Marmot Creek Research Basin from description by Fisera (1977).

\begin{tabular}{ll}
\hline Snow course & Description \\
\hline 1 & East-sloping lodgepole pine about $9 \mathrm{~m}$ tall with natural openings \\
3 & Gently south-sloping mature spruce, lodgepole pine, and alpine \\
6 & Gently northeast-sloping mature spruce, lodgepole pine, and alpine fir \\
8 & South sloping lodgepole pine about $6 \mathrm{~m}$ tall \\
11 & Southeast-sloping mature spruce, lodgepole pine, and alpine fir \\
14 & Northeast-sloping mature spruce, lodgepole pine, and alpine fir with small natural openings \\
19 & Variable terrains (i.e. north and south slope, flat, and gullies) above treeline \\
\hline
\end{tabular}

Table 6. Active groundwater wells (GWs) at the Marmot Creek Research Basin.

\begin{tabular}{lllrrll}
\hline GW well & Station name & Established & Elevation (m) & Depth (m) & Aquifer & Lithology \\
\hline 301 & Marmot Creek Basin S5250_0301 & 11 October 1964 & 1601.4 & 12.2 & Rocky Mountain & Sandstone \\
303 & Marmot Creek Basin N5475_0303 & 9 July 1965 & 1669.1 & 36.58 & Rocky Mountain & Sandstone \\
305 & Marmot Creek Basin N6770_0305 & 14 July 1965 & 2063 & 11.58 & Fernie & Shale \\
386 & Marmot Creek Basin N2507E_0386 & 18 November 1988 & 1894 & 12.8 & Surficial & Gravel and clay \\
\hline
\end{tabular}

\section{Groundwater data}

Three groundwater wells (GWs), 301, 303, and 305, established in the 1960s and one GW, 386, established in 1988 are continuously monitored by AEP. The location of these groundwater wells is shown in Fig. 1, and brief information regarding these wells is provided in Table 6 . Daily data for these groundwater wells can be searched and accessed from AEP's "Groundwater Observation Well Network (GOWN)" website at http://environment.alberta.ca/ apps/GOWN/. Access to the hourly groundwater well data can be requested from the Groundwater Information Centre at gwinfo@gov.ab.ca.

\section{Example data}

Data from the June 2013 flood are shown as an example of weather and streamflow observed in MCRB (Fig. 11). The flood event started on 18 June and ended on 24 June. Air temperature observed at the Fisera Ridge station was as high as $8^{\circ} \mathrm{C}$ during rainfall on $19 \mathrm{June}$ and dropped to $0.4^{\circ} \mathrm{C}$ during snowfall on 21 June; the atmosphere became saturated on 18 June and stayed saturated through 21 June (Fig. 11a). Variable wind speeds were observed at the Fisera Ridge station, changing from relatively calm conditions on 18 June to $4 \mathrm{~m} \mathrm{~s}^{-1}$ on 20 June, then dropping to an average of $2 \mathrm{~m} \mathrm{~s}^{-1}$ before peaking at $5.5 \mathrm{~m} \mathrm{~s}^{-1}$ on 21 June (Fig. 11b). Overcast skies persisted during much of the flood event and incoming solar radiation observed at the Fisera Ridge station dropped from a peak of $533 \mathrm{~W} \mathrm{~m}^{-2}$ on 18 June to below $266 \mathrm{~W} \mathrm{~m}^{-2}$ throughout the event and then rose to a peak of $1038 \mathrm{~W} \mathrm{~m}^{-2}$ on 22 June (Fig. 11b). Similar depths of precipitation fell at all elevations (1436 to $2325 \mathrm{~m}$ ) in MCRB, with about $257 \mathrm{~mm}$ during 19-25 June; however, this measurement was compromised as the Geonor precipitation gauge was overtopped on 21 June and could not be immediately accessed for maintenance due to damaged trails and roads. During the snowfall of 21-22 June, the depth of fresh snowpack on the ground was used to estimate precipitation based on the assumption of a fresh snow density of $100 \mathrm{~kg} \mathrm{~m}^{-3}$ (Pomeroy et al. 2016). Approximately $237 \mathrm{~mm}$ of rainfall was measured at the Fisera Ridge station during 19-25 June, and an $8 \mathrm{~cm}$ deep snowpack developed at Fisera Ridge on 21 June and melted after 22 June (Fig. 11c). Rainfall and snowfall rates during the event remained less than $12 \mathrm{~mm} \mathrm{~h}^{-1}$ and were higher than $6 \mathrm{~mm} \mathrm{~h}^{-1}$ only on 19 and 20 June, with cumulative daily totals increasing from $41 \mathrm{~mm}$ on 19 June to $113 \mathrm{~mm}$ on 20 June, and then dropping to 77 and $18 \mathrm{~mm}$ on 21 and 22 June, respectively. The streamflow discharge observed at the outlet of Upper Marmot Creek remained below $0.6 \mathrm{~mm} \mathrm{~h}^{-1}$ at the start of the flood event on 19 June and increased steadily on 20 June, reaching a peak of $2.84 \mathrm{~mm} \mathrm{~h}^{-1}$ at 01:00 on 21 June and then falling to below $1 \mathrm{~mm} \mathrm{~h}^{-1}$ after 21 June for the remainder of the flood event (Fig. 11d). Total discharge generated at the outlet of Upper Marmot Creek was estimated to be $106 \mathrm{~mm}$ during 19-25 June, much of which was the result of rain on snow in the alpine and treeline elevations.

\section{Compilation of Marmot Creek memories, real-time data, and publications}

The Centre for Hydrology held a 50th Anniversary Workshop for MCRB in February 2013 where many of the original and recent researchers gave presentations on a halfcentury of scientific research in the basin. The centre has also compiled 120 MCRB publications and provides realtime observations from many of the current meteorological stations. The workshop presentations, publications, and 

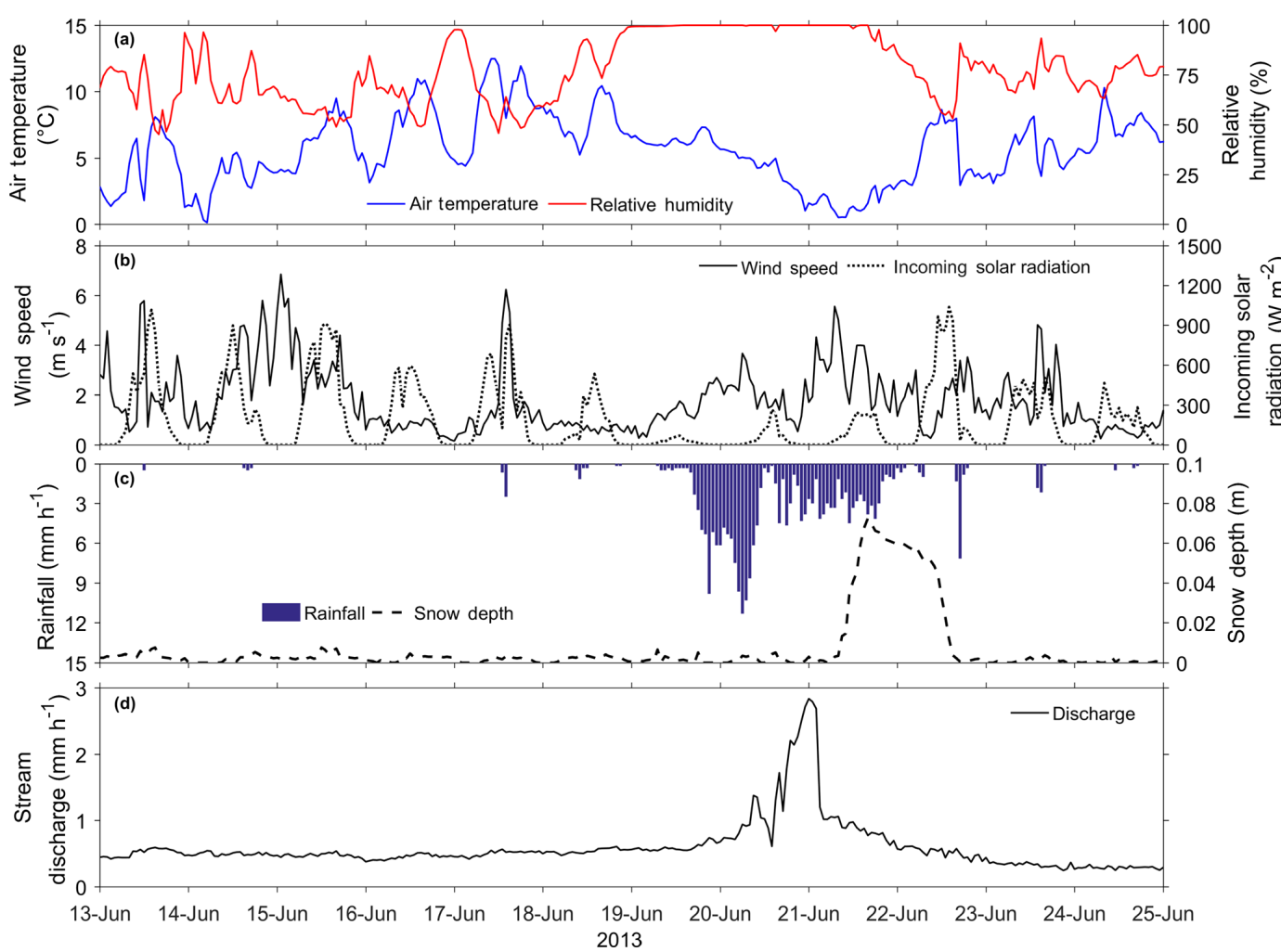

Figure 11. Example of hourly-averaged observations during 13-25 June 2013 from the Fisera Ridge station at the Marmot Creek Research Basin showing (a) air temperature and relative humidity, (b) wind speed and incoming solar radiation, (c) rainfall and snow depth, and (d) stream discharge from Upper Marmot Creek.

data can be accessed here: http://www.usask.ca/hydrology/ MarmotBasin.php. In addition, a number of recent theses contain detailed contemporary site information for Marmot Creek Research Basin and provide results for the recent research conducted in the basin. These theses can help familiarise researchers with the basin and better understand its hydrology. Table 7 lists the theses that can be searched and accessed from the University of Saskatchewan's "eCommons" website at https://ecommons.usask.ca/.

\section{Data availability}

All data presented in this paper are publicly available at the Federated Research Data Repository (https://doi.org/10.20383/101.09, Fang et al., 2018). Headers in most data files are self-explanatory, and all data are measured in central standard time (CST) that is $6 \mathrm{~h}$ behind Greenwich mean time (GMT -6$)$. Meteorological data are time series in comma-delimited txt files organised by station. Snow survey data are stored in the .xlsx files. Historical snow survey data are summarised in a single time series file. Recent snow survey data are organised by site for a water year. Recent streamflow data are time series and are stored in .csv files and are organised by the gauge station. Additional readme files are provided for notes on missing data, data measurement periods and units, and no measurement due to wildlife interruption. Additional GIS shapefiles are provided to show locations of historical and recent hydrometeorological and hydrometric stations as well as historical and recent snow survey transects.

\section{Summary}

Data presented in this paper provide support to ongoing research in MCRB, a mountain basin located in the Front Range of the Canadian Rockies. The data include 11 water years of hourly gap-filled air temperature, relative humidity, wind speed, precipitation, incoming solar radiation, and soil temperature from 1 October 2005 to 30 September 2016 as well as 18 water years of hourly air temperature, relatively humidity, and wind speed as well as daily precipitation from 1 October 1969 to 30 September 1987. These meteorological datasets are useful for driving hydrological models and carrying out diagnostic change detection analysis in the basin. In addition, 15 min quality-controlled data including other hydrometeorological variables such as snow depth, soil temperature, and soil moisture are presented from 1 October 2005 to 30 September 2016; these data have gaps but are useful for diagnosing model performance in snow accumulation, soil moisture, and temperature. Snow survey data are included 
Table 7. Marmot Creek Research Basin theses in chronological order for the recent period.

\begin{tabular}{|c|c|c|}
\hline Thesis title & Author & Year \\
\hline Energy fluxes at the air-snow interface & Helgason, Warren & 2009 \\
\hline Hydrological response unit-based blowing snow modelling over mountainous terrain & MacDonald, Matthew & 2010 \\
\hline Radiation and snowmelt dynamics in mountain forests & Ellis, Chad & 2011 \\
\hline Simulating areal snowcover depletion and snowmelt runoff in alpine terrain & DeBeer, Chris & 2012 \\
\hline Acoustic measurement of snow & Kinar, Nicholas & 2013 \\
\hline Effects of climate variability on hydrological processes in a Canadian Rockies headwaters catchment & Siemens, Evan & 2016 \\
\hline Sensitivity analysis of mountain hydrology to changing climate & Rasouli, Kabir & 2017 \\
\hline
\end{tabular}

for the historical period from 1963 to 1986 and the current period from 2007 to 2016. Hourly streamflow is provided for Cabin, Middle, and Twin Creeks from 2007 to 2012, Upper Marmot Creek from 2007 to 2016, and Marmot Creek after June 2013 flood from 26 June 2013 to 2016. Daily streamflow for Cabin Creek, Middle Creek, Twin Creek, and Upper Marmot Creek from 1963 to 1986 and Marmot Creek daily streamflow from 1962 to 19 June 2013 can be obtained from the ECCC Water Survey of Canada's "historical hydrometric data search" website. In addition, data from several groundwater wells in Marmot Creek can be accessed from AEP's "Groundwater Observation Well Network (GOWN)" website. In all, these long-term meteorological and hydrometric datasets are a legacy of previous and current research activities conducted in MCRB and support ongoing efforts to detect and diagnose climate change in the basin and region, examine extreme hydrometeorological events (i.e. drought and flood), and diagnose the basin response to land cover changes caused by stressors such as insect infestations, fire, and forest harvesting. This dataset ultimately serves to advance our knowledge of hydrology of the Canadian Rockies. 
Appendix A: Abbreviation list

$\begin{array}{ll}\text { AEP } & \text { Alberta Environment and Parks } \\ \text { a.g.s. } & \text { above ground surface } \\ \text { AWRP } & \text { Alberta Watershed Research Program } \\ \text { CCG } & \text { Cabin Creek gauge } \\ \text { CFS } & \text { Canadian Forestry Service } \\ \text { CRHM } & \text { Cold Regions Hydrological Modelling platform } \\ \text { CST } & \text { central standard time } \\ \text { DEM } & \text { digital elevation model } \\ \text { ECCC } & \text { Environment and Climate Change Canada } \\ \text { GIS } & \text { geographic information system } \\ \text { GOWN } & \text { Groundwater Observation Well Network } \\ \text { GW } & \text { groundwater wells } \\ \text { Lidar } & \text { light detection and ranging } \\ \text { MCG } & \text { Middle Creek gauge } \\ \text { MCRB } & \text { Marmot Creek Research Basin } \\ \text { MSC } & \text { Meteorological Service of Canada } \\ \text { QC } & \text { quality controlled } \\ \text { ROC } & \text { rate of change } \\ \text { SC } & \text { snow courses } \\ \text { SRB } & \text { Saskatchewan River basin } \\ \text { SWE } & \text { snow water equivalent } \\ \text { TCG } & \text { Twin Creek gauge } \\ \text { UMCG } & \text { Upper Marmot Creek gauge } \\ \text { WY } & \text { water year }\end{array}$


Author contributions. XF cleaned and organised the dataset. JWP designed and instrumented the research basin, and all authors collected data and contributed to the paper writing.

Competing interests. The authors declare that they have no conflict of interest.

Special issue statement. This article is part of the special issues "Hydrometeorological data from mountain and alpine research catchments" and "Water, ecosystem, cryosphere, and climate data from the interior of western Canada and other cold regions". It is not associated with a conference.

Acknowledgements. The authors would like to gratefully acknowledge the funding assistance provided from the Alberta government departments Environment and Parks, and Agriculture and Forestry, the IP3 Cold Regions Hydrology Network of the Canadian Foundation for Climate and Atmospheric Sciences, the Natural Sciences and Engineering Research Council of Canada through Discovery Grants, Research Tools and Instrument Grants, Alexander Graham Bell Scholarships, and the Changing Cold Regions Network, the Global Institute for Water Security, Global Water Futures and the Canada Research Chairs programme. Logistical assistance was received from the Biogeoscience Institute, University of Calgary and the Nakiska Ski Area. Field work by many graduate students in and collaborators with the Centre for Hydrology and research officers Michael Solohub, May Guan, Angus Duncan, and Greg Galloway was essential in accurate data collection in adverse conditions. Natural Resources Canada, Canadian Forest Service are the owners of the copyright of the historical meteorological and snow survey data. This paper is dedicated to the hundreds of researchers who have contributed to data collection in Marmot Creek over the last 55 years.

Review statement. This paper was edited by Danny Marks and reviewed by two anonymous referees.

\section{References}

Alberta Forest Service: Marmot Creek Watershed Research Basin: Forest Cover Type Map, Information and Technical Services Division, Graphics Section, Department of Forestry of Canada, Ottawa, Ontario, 1963.

Beke, G. J.: Soils of three experimental watersheds in Alberta and their hydrological significance, $\mathrm{PhD}$ thesis, Department of Soil Science, University of Alberta, Edmonton, Alberta, Canada, 456 pp., 1969.

Bruce, J. P. and Clark, R. H.: Introduction to Hydrometeorology, Pergamon Press, Toronto, Canada, 319 pp., 1965.

Canadian Forestry Service: Compilation of hydrometeorological record, Marmot Creek Basin, 1976 data, Volume 12, Northern Forest Research Centre, Edmonton, Alberta, 1976.
DeBeer, C. M. and Pomeroy, J. W.: Modelling snow melt and snowcover depletion in a small alpine cirque, Canadian Rocky Mountains, Hydrol. Process., 23, 2584-2599, https://doi.org/10.1002/hyp.7346, 2009.

DeBeer, C. M. and Pomeroy, J. W.: Influence of snowpack and melt energy heterogeneity on snow cover depletion and snowmelt runoff simulation in a cold mountain environment, J. Hydrol., 553, 199-213, https://doi.org/10.1016/j.jhydrol.2017.07.051, 2017.

Ellis, C. R. and Pomeroy, J. W.: Estimating sub-canopy shortwave irradiance to melting snow on forested slopes, Hydrol. Process., 21, 2581-2593, https://doi.org/10.1002/hyp.6794, 2007.

Ellis, C. R., Pomeroy, J. W., Essery, R. L. H., and Link, T. E.: Effects of needleleaf forest cover on radiation and snowmelt dynamics in the Canadian Rocky Mountains, Can. J. Forest Res., 41, 608620, https://doi.org/10.1139/X10-227, 2011.

Ellis, C. R., Pomeroy, J. W., and Link, T. E.: Modeling increases in snowmelt yield and desynchronization resulting from forest gap thining treatments in a northern mountain catchment, Water Resour. Res., 49, 936-949, https://doi.org/10.1002/wrcr.20089, 2013.

Essery, R., Pomeroy, J., Ellis, C., and Link, T.: Modelling longwave radiation to snow beneath forest canopies using hemispherical photography or linear regression, Hydrol. Process., 22, 27882800, https://doi.org/10.1002/hyp.6930, 2008.

Fang, X. and Pomeroy, J. W.: Impact of antecedent conditions on simulations of a flood in a mountain headwater basin, Hydrol. Process., 30, 2754-2772, https://doi.org/10.1002/hyp.10910, 2016.

Fang, X., Pomeroy, J. W., Ellis, C. R., MacDonald, M. K., DeBeer, C. M., and Brown, T.: Multi-variable evaluation of hydrological model predictions for a headwater basin in the Canadian Rocky Mountains, Hydrol. Earth Syst. Sci., 17, 1635-1659, https://doi.org/10.5194/hess-17-1635-2013, 2013.

Fang, X., Pomeroy, J., DeBeer, C., Harder, P., and Siemens, E.: Hydrometeorological data from Marmot Creek Research Basin, Canadian Rockies, Federated Research Data Repository (FRDR), https://doi.org/10.20383/101.09, 2018.

Fisera, Z.: Snow accumulation and melt pattern in tree line stands of Marmot Creek Basin, in: Proceedings of Alberta Watershed Research Program Symposium, Information Report NOR-X-176, Canadian Forestry Service, Northern Forestry Centre, Edmonton, Alberta, 97-109, 1977.

Golding, D. L. and Swanson, R. H.: Snow distribution patterns in clearings and adjacent forest, Water Resour. Res., 22, 19311940, 1986.

Harder, P. and Pomeroy, J. W.: Estimating precipitation phase using a psychrometric energy balance method, Hydrol. Process., 27, 1901-1914, https://doi.org/10.1002/hyp.9799, 2013.

Harder, P., Pomeroy, J. W., and Westbrook, C. J.: Hydrological resilience of a Canadian Rockies headwaters basin subject to changing climate, extreme weather, and forest management, Hydrol. Process., 29, 3905-3924, https://doi.org/10.1002/hyp.10596, 2015.

Harlan, R. L.: Soil-water freezing, snow accumulation and ablation in Marmot Creek Experimental Watershed, Alberta, Canada, in: Proceedings of the 37th Western Snow Conference, 15-17 April 1969, Salt Lake City, Utah, 29-33, 1969. 
Hillman, G. R. and Golding, D. L.: Forest floor characteristics of Marmot and Streeter experimental watersheds, Alberta, Information Report NOR-X-234, Canadian Forestry Service, Northern Forestry Centre, Edmonton, 22 pp., 1981.

Hopkinson, C., Pomeroy, J. W., DeBeer, C., Ellis, C., and Anderson, A.: Relationships between snowpack depth and primary LiDAR point cloud derivatives in a mountainous environment, in: Remote Sensing and Hydrology, Jackson Hole, Wyoming, USA, September 2010, IAHS Press, IAHS Publ. 352, 2012.

Jeffrey, W. W.: Experimental watersheds in the Rocky Mountains, Alberta, Canada, in: Symposium of Budapest, Proceedings of the Symposium on Representative and Experimental Areas, 28 September-5 October 1965, Budapest, Hungary, 502-521, 1965.

Keith, D. M., Johnson, E. A., and Valeo, C.: A hillslope forest floor (duff) water budget and the transition to local control, Hydrol. Process., 24, 2738-2751, https://doi.org/10.1002/hyp.7697, 2010.

Kinar, N. J. and Pomeroy, J. W.: Automated determination of snow water equivalent by acoustic reflectometry, IEEE T. Geosci. Remote., 47, 3161-3167, https://doi.org/10.1109/TGRS.2009.2019730, 2009.

Kinar, N. J. and Pomeroy, J. W.: SAS2: the system for acoustic sensing of snow, Hydrol. Process., 29, 4032-4050, https://doi.org/10.1002/hyp.10535, 2015.

Kirby, C. L. and Ogilvy, R. T.: The forest of Marmot Creek watershed research basin, Canadian Forestry Service Publication No. 1259, Canadian Department of Fisheries and Forestry, Ottawa, Ontario, Canada, 37 pp., 1969.

MacDonald, M. K., Pomeroy, J. W., and Pietroniro, A.: On the importance of sublimation to an alpine snow mass balance in the Canadian Rocky Mountains, Hydrol. Earth Syst. Sci., 14, 14011415, https://doi.org/10.5194/hess-14-1401-2010, 2010.

Marsh, C. B., Pomeroy, J. W., and Spiteri, R. J.: Implications of mountain shading on calculating energy for snowmelt using unstructured triangular meshes, Hydrol. Process., 26, 1767-1778, https://doi.org/10.1002/hyp.9329, 2012.

Munn, R. E. and Storr, D.: Meteorological studies in the Marmot Creek Watershed, Alberta, Canada, in August 1965, Water Resour. Res., 3, 713-722, 1967.

Musselman, K. N. and Pomeroy, J. W.: Estimation of neeedleleaf canopy and trunk temperatures and longwave contribution to melting snow, J. Hydrometeorol., 18, 555-572, https://doi.org/10.1175/JHM-D-16-0111.1, 2017.

Neill, C. R.: Forest management for increased water yield - how used in southern Alberta?, Can. Water Resour. J., 5, 56-75, https://doi.org/10.4296/cwrj0501056, 1980.

Pomeroy, J. W., Marks, D., Link, T., Ellis, C., Hardy, J., Rowlands, A., and Granger, R.: The impact of coniferous forest temperature on incoming longwave radiation to melting snow, Hydrol. Process., 23, 2513-2525, https://doi.org/10.1002/hyp.7325, 2009.
Pomeroy, J., Fang, X., and Ellis, C.: Sensitivity of snowmelt hydrology in Marmot Creek, Alberta, to forest cover disturbance, Hydrol. Process., 26, 1892-1905, https://doi.org/10.1002/hyp.9248, 2012.

Pomeroy, J. W., Fang, X., and Rasouli, K.: Sensitivity of snow processes to warming in the Canadian Rockies, in: Proceedings of the 72nd Eastern Snow Conference, 9-11 June 2015, Sherbrooke, Québec, Canada, 22-33, 2015.

Pomeroy, J. W., Fang, X., and Marks, D.: The cold rain-onsnow event of June 2013 in the Canadian Rockies - characteristics and diagnosis, Hydrol. Process., 30, 2899-2914, https://doi.org/10.1002/hyp.10905, 2016.

Redmond, D. R.: Organization of inter-agency watershed research programs for Canada, in: Proceedings of 4th Hydrology Symposium on Research Watersheds, Guelph, Ontario, Canada, 299304, 1 May 1964.

Rothwell, R., Hillman, G., and Pomeroy, J. W.: Marmot Creek Experimental Watershed Study, Forest. Chron., 92, 32-36, https://doi.org/10.5558/tfc2016-010, 2016.

Siemens, E.: Effects of climate variability on hydrological processes in a Canadian Rockies headwaters catchment, MSc thesis, Department of Geography and Planning, University of Saskatchewan, Saskatoon, Saskatchewan, 71 pp., 2016.

Smith, C. D.: Correcting the wind bias in snowfall measurements made with a Geonor T-200B precipitation gauge and alter wind shield, in: Proceedings of the 87th American Meteorology Society Annual Meeting, San Antonio, Texas, 13-18 January 2007.

Stevenson, D. R.: Geological and groundwater investigations in the Marmot Creek experimental basin of southwestern Alberta, Canada, MSc thesis, Department of Geology, University of Alberta, Edmonton, Alberta, 106 pp., 1967.

Storr, D.: Precipitation variations in a small forested watershed, in: Proceedings of the 35th Western Snow Conference, 18-20 April 1967, Boise, Idaho, United States, 11-17, 1967.

Storr, D.: Wind-snow relations at Marmot Creek, Alberta, Can. J. Forest Res., 3, 479-485, 1973.

Swanson, R. H. and Golding, D. L.: Snowpack management on Marmot watershed to increase late season streamflow, in: Proceedings of the 50th Western Snow Conference, 20-23 April 1982, Reno, Nevada, 215-218, 1982.

Swanson, R. H., Golding, D. L., Rothwell, R. L., and Bernier, P. Y.: Hydrologic effects of clear-cutting at Marmot Creek and Streeter Watersheds, Alberta, Canadian Forestry Service, Northern Forestry Centre, Edmonton, Information Report NOR-X278, 27 pp., 1986. 